\title{
Degree Lower Bounds of Tower-Type for Approximating Formulas with Parity Quantifiers*
}

\author{
Albert Atserias $^{\dagger}$ \\ Universitat Politècnica de Catalunya \\ Anuj Dawar ${ }^{\ddagger}$ \\ University of Cambridge
}

June 25, 2013

\begin{abstract}
Kolaitis and Kopparty have shown that for any first-order formula with parity quantifiers over the language of graphs there is a family of multi-variate polynomials of constant-degree that agree with the formula on all but a $2^{-\Omega(n)}$-fraction of the graphs with $n$ vertices. The proof bounds the degree of the polynomials by a tower of exponentials whose height is the nesting depth of parity quantifiers in the formula. We show that this tower-type dependence is necessary. We build a family of formulas of depth $q$ whose approximating polynomials must have degree bounded from below by a tower of exponentials of height proportional to $q$. Our proof has two main parts. First, we adapt and extend the results by Kolaitis and Kopparty that describe the joint distribution of the parities of the numbers of copies of small subgraphs in a random graph to the setting of graphs of growing size. Second, we analyse a variant of Karp's graph canonical labeling algorithm and exploit its massive parallelism to get a formula of low depth that defines an almost canonical pre-order on a random graph.
\end{abstract}

\section{Introduction}

Since the 0-1 law for first-order logic was established [5, 6], there has been much interest in exploring the asymptotic properties of definable classes of graphs. Many extensions of first-order logic have been shown to have a 0-1 law (see for instance [11, 4]) and in many other cases, weaker forms of convergence have been established (see [3]). A recent, remarkable result in this vein is that of Kolaitis and Kopparty [9] who study $\mathrm{FO}[\oplus]$, the extension of first-order logic with parity quantifiers. They show that for every constant edge-probability $p$ and for every $\mathrm{FO}[\oplus]$-sentence $\phi$, there are two explicitly computable rational numbers $a_{0}, a_{1}$ such that, for $i \in\{0,1\}$, as $n$ approaches infinity, the probability that the random graph $G(2 n+i ; p)$ satisfies $\phi$ approaches $a_{i}$. In other words, $\phi$ has an asymptotic probability $a_{0}$ on the sequence of graphs of even cardinality and $a_{1}$ on the sequence of those of odd cardinality. The proof of this result brings entirely new methods to the analysis of the asymptotic behaviour of logics on graphs, based on discrete analysis and polynomials over finite fields. In particular, it ties this to the study of approximations of circuits by low-degree polynomials, as we explain next.

The 0-1 law for first-order logic, in its general form is a quantifier-elimination result. It states that for any first-order formula $\phi$, there is a quantifier-free formula $\theta$ such that $\phi$ is equivalent to $\theta$ almost surely.

\footnotetext{
*A preliminary shorter version of this paper appeared in the Proceedings of 39th International Colloquium on Automata, Languages and Programming (ICALP), Part II, Lecture Notes in Computer Science 7392, Springer, Warwick, UK, July 9-13, 2012.

${ }^{\dagger}$ Research of first author partially supported by CICYT TIN2010-20967-C04-04 (TASSAT).

${ }^{\ddagger}$ Research of second author partially supported by EPSRC grant EP/H026835.
} 
To be precise, $\phi$ and $\theta$ agree on a fraction $1-2^{-\epsilon n}$ of the graphs on $n$ vertices. We can say that any first-order formula is well approximated by a quantifier-free formula. This is similar to the phenomenon of depth-reduction for circuits which has a long history in computational complexity theory. For instance, Allender showed that $\mathrm{AC}^{0}$-circuits have equivalent $\mathrm{TC}^{0}$-circuits of depth 3 and quasi-polynomial size [1]. The result of Beigel and Tarui that general $\mathrm{ACC}^{0}$-circuits have equivalent depth- 2 circuits of quasi-polynomial size with a symmetric gate at the root [2] has been exploited to remarkable effect recently in the work of Williams [14]. In the context of approximation, one of the best known examples is the Razborov-Smolensky approximation of $\mathrm{AC}^{0}[\oplus]$-circuits by multi-variate polynomials over $\mathbb{Z}_{2}$ of polylogarithmic degree $[12,13]$. The method yields an approximation that agrees on a fraction $1-2^{-(\log n)^{c}}$ of the inputs.

The Kolaitis-Kopparty result mentioned above is proved by a depth-reduction argument of a similar kind that exploits the higher degree of symmetry that $\mathrm{FO}[\oplus]$-formulas have over $\operatorname{AC}^{0}[\oplus]$-circuits. They prove that every $\mathrm{FO}[\oplus]$-formula $\phi$ is well-approximated by a formula which is a Boolean combination of quantifier-free formulas and of polynomials over $\mathbb{Z}_{2}$ of special form, that we call $F O[\oplus]$-polynomials, and the degree of these polynomials depends only on $\phi$. These polynomials have as variables $X_{u v}$ for every potential edge $\{u, v\}$ over the vertex-set $\{1, \ldots, n\}$. For example, the $\mathrm{FO}[\oplus]$-polynomial that gives the parity of the number of triples that extend the vertex $u$ to a triangle is

$$
\sum_{\substack{v: \\ v \neq u}} \sum_{\substack{w: \\ w \neq u \\ w \neq v}} X_{u v} X_{v w} X_{w u} .
$$

At the heart of the argument is the analysis of the bias of certain low degree polynomials of this type on uniformly random inputs. This understanding is then used to carry over a quantifier-elimination argument that eliminates one parity quantifier or one first-order quantifier at a time. Relevant to our work is the fact that, intriguingly, the elimination of each parity quantifier in this argument incurs an exponential loss in the degree. The final outcome is that the degree $d$ of the approximating polynomials is bounded from below by a function of tower-type on the number $q$ of parity quantifiers that were eliminated, i.e.

$$
d \geq 2^{2^{2}}
$$

where the height of the tower is at least $q$. At first sight, the source of this inefficiency in the proof appears technical and it might be tempting to think that a different method could perhaps avoid it altogether.

In this paper we prove that the non-elementary dependence stated in equation (2) cannot be avoided. To be precise, we construct an explicit family of $\mathrm{FO}[\oplus]$-formulas $\phi_{q}$ of quantifier rank $q$ and prove that they cannot be approximated by a Boolean combination of quantifier-free formulas and polynomials of degree bounded by an elementary function of $q$. Specifically, we prove the following:

Theorem 1. There exists a constant $c>0$ such that for every large enough integer $q$, every $\epsilon>0$, and every large enough integer $n$, there exists an $F O[\oplus]$-formula $\phi(u, v, w)$ of quantifier rank $q$ such that, for every Boolean combination $p$ of quantifier-free formulas and $F O[\oplus]$-polynomials of degree bounded by a tower of exponentials of height at most $q / c$, the formulas $\phi$ and $p$ must disagree on a fraction $1-\epsilon$ of all graphs with $n$ vertices.

By an $\mathrm{FO}[\oplus]$-polynomial we mean a formula that has a direct translation to a bounded-degree polynomial over $\mathbb{Z}_{2}$ : a sequence of parity quantifiers followed by a conjunction of atomic facts. Since both $\phi$ and $p$ have free variables, when we say that $\phi$ and $p$ disagree on a graph $G$, we mean that the set of tuples of vertices of $G$ satisfying $\phi$ is not the same as the set of tuples satisfying $p$. 
Theorem 1 should be contrasted with the 0-1 law for first-order logic. In that case the approximating formula is quantifier-free, and such formulas translate into polynomials of degree at most polynomial in the number of free variables.

Proof outline and techniques Our proof relies on two technical ingredients. On one hand we analyse a canonical labeling algorithm for graphs due to Karp [8] (see [7] for another view on the logical definability of Karp's canonical labeling). We exploit its massive parallelism to build an $\mathrm{FO}[\oplus]$-formula $\psi(u, v)$ of depth $O\left(\log ^{*} n\right)$ that works on graphs with $n$ vertices. The formula is designed in such a way that, on almost every graph, it defines a linear pre-order of width at most two on the set of vertices of the graph. The second ingredient is a refined analysis of one of the key tools from the Kolaitis-Kopparty paper. Using and extending their techniques for estimating the frequencies mod 2 of subgraph copies, we show that for every $\mathrm{FO}[\oplus]$-polynomial $p(u, v, w)$ of degree $\log \log \log n$ and for the random graph $G(n, 1 / 2)$, with high probability there exists a triple of distinct vertices $(a, b, c)$ such that $p$ cannot distinguish it from any of its permutations.

From these two ingredients, the lower bound follows by taking the formula $\phi(u, v, w):=\psi(u, v) \wedge$ $\psi(v, w)$. On one hand this formula distinguishes at least one permutation of the vertices $(a, b, c)$ from some other. This is because by linearity of the pre-order the classes they lie in must be comparable, but by the width-2 condition on the pre-order not all three vertices can sit in the same class. On the other hand, if $\phi^{\prime}(u, v, w)$ is any Boolean combination of quantifier-free formulas and $\mathrm{FO}[\oplus]$-polynomials of degree $\log \log \log n$, we could choose $(a, b, c)$ in such a way that $\phi^{\prime}$ is not able to distinguish any permutation of $(a, b, c)$ from the others. We conclude that $\phi^{\prime}$ cannot approximate $\phi$, and since the quantifier rank of $\phi$ is still $O\left(\log ^{*} n\right)$, the tower-type lower bound follows. We provide more details in the body of the paper.

Section 2 below introduces some essential notation. Then, in Section 3 we show that for any Boolean combination of polynomials of low-degree, on a sufficiently large random graph, there is some tuple of elements which is not distinguished from any of its permutations. Section 4 contains the construction of the formula $\psi(u, v)$ that defines a linear pre-order of width at most two on almost all graphs on $n$ vertices. Finally, Section 5 pulls these ingredients together to establish our result. The construction in Section 3 relies heavily on elements from [9] and [10]. Similarly, Section 4 borrows from the analysis in [8]. The reader wishing to follow all details of the proof may wish to have copies of these three papers at hand.

\section{Preliminaries}

We use $[n]$ to denote the set $\{1, \ldots, n\}$. We identify the nodes of a complete rooted binary tree with the binary strings that start with the symbol 1: the root is 1 , the left child of $t$ is $t 0$ and the right child of $t$ is $t 1$. The level-order of a complete binary tree is $1,10,11,100,101,110,111,1000,1001, \ldots$, i.e. the nodes are ordered first by length, and within each length, they are ordered lexicographically. Note that if the strings are interpreted as numbers written in binary, this is the usual order of the natural numbers. For a natural number $n \geq 1$, we write $\operatorname{bin}_{2}(n)$ for its unique binary encoding with a leading one.

Let $G$ and $H$ be graphs. We write $V(G)$ and $E(G)$ to denote the vertices and edges of $G$ respectively, and similarly for $H$. A homomorphism from $G$ to $H$ is a mapping $h: V(G) \rightarrow V(H)$ that maps edges to edges; i.e. such that if $\{u, v\} \in E(G)$, then $\{h(u), h(v)\} \in E(H)$. Let $\operatorname{Hom}(G, H)$ denote the collection of all homomorphisms from $G$ to $H$.

The collection of $\mathrm{FO}[\oplus]$-formulas over the language of graphs is the smallest class of formulas that contains the atomic formulas $E(x, y)$ and the equalities $x=y$, and is closed under negation, conjunction and disjunction, universal and existential quantification, and parity quantification; i.e. quantification of the 
form $\oplus x \phi(x)$. The meaning of $\oplus x \phi(x)$ is that there is an odd number of vertices $x$ that satisfy $\phi(x)$. For a tuple $\mathbf{a}=\left(a_{1}, \ldots, a_{k}\right)$ and a permutation $\pi \in S_{k}$, we write $\mathbf{a} \circ \pi$ for the tuple $\left(a_{\pi(1)}, \ldots, a_{\pi(k)}\right)$. If $p\left(x_{1}, \ldots, x_{k}\right)$ is a formula with free variables $x_{1}, \ldots, x_{k}$, and $y_{1}, \ldots, y_{k}$ are variables or constants, we write $p\left(y_{1}, \ldots, y_{k}\right)$ for the result of replacing each occurrence of $x_{i}$ by $y_{i}$. This applies also to the case where $y_{1}, \ldots, y_{k}$ is a permutation of $x_{1}, \ldots, x_{k}$.

An atomic type on the variables $x_{1}, \ldots, x_{k}$ over the language of graphs is a consistent collection of atomic formulas $E\left(x_{i}, x_{j}\right)$ or $x_{i}=x_{j}$ and negated atomic formulas $\neg E\left(x_{i}, x_{j}\right)$ or $x_{i} \neq x_{j}$ that is maximal with respect to set-inclusion. A positive atomic type is the subset of an atomic type containing all its positive atomic formulas. We say that a type is injective if it contains the formula $x_{i} \neq x_{j}$ whenever $i \neq j$. An equality type is the subset of an atomic type containing all its equalities $x_{i}=x_{j}$ and inequalities $x_{i} \neq x_{j}$. For a graph $G$ and a tuple $\mathbf{a}=\left(a_{1}, \ldots, a_{k}\right) \in V(G)^{k}$, the atomic type of $\mathbf{a}$ in $G$ is the unique atomic type that is made true in $G$ by the assignment $x_{i} \mapsto a_{i}$. The atomic type of $a_{k}$ over $\left(a_{1}, \ldots, a_{k-1}\right)$ in $G$ is the subset of the atomic type of a in $G$ containing all formulas that involve the variable $x_{k}$.

\section{Fooling polynomials of low degree}

In this section we aim to establish that for any $\mathrm{FO}[\oplus]$-formula which is a Boolean combination of polynomials of low degree (growing as $O(\log \log \log n)$ ) and a sufficiently large random graph $G$, with high probability there is a triple of vertices $a, b, c$ such that the formula does not distinguish this triple from any of its permutations. To do this, we first establish a normal form for such $\mathrm{FO}[\oplus]$-formulas which will permit an analysis of their asymptotic behaviour. The normal form is established in Section 3.3, and the analysis in Section 3.4.

Once we have the normal form, the proof strategy is roughly as follows. Fix a formula in normal form $p(x, y, z)$. For every fixed $a, b, c \in[n]$, let $Y(a, b, c)$ be the event that $p$ cannot distinguish any two permutations of $a, b, c$. Ideally we would like to show that the event $Y(a, b, c)$ has non-negligible probability of happening, and that if $a^{\prime}, b^{\prime}, c^{\prime} \in[n]$ is a triple disjoint from $a, b, c$, then the events $Y(a, b, c)$ and $Y\left(a^{\prime}, b^{\prime}, c^{\prime}\right)$ are almost independent. If we were able to do this, the result would follow from an application of Chebyshev's inequality. Unfortunately it is not quite true that $Y(a, b, c)$ and $Y\left(a^{\prime}, b^{\prime}, c^{\prime}\right)$ are almost independent in general, so we need to take a detour. The detailed argument is given in Section 3.5

\subsection{Formulas and polynomials}

In this section, we define the formulas to which our result applies. In short, they are Boolean combinations of $\mathrm{FO}[\oplus]$-polynomials. An $\mathrm{FO}[\oplus]$-polynomial is a formula of $\mathrm{FO}[\oplus]$ consisting of a sequence of parity quantifiers followed by a conjunction of atomic formulas and negated equalities. In its general form, an $\mathrm{FO}[\oplus]$-polynomial $p$ with free variables $u_{1}, \ldots, u_{k}$ is a formula of the form

$$
\oplus u_{k+1} \cdots \oplus u_{m}\left(\bigwedge_{i \neq j} u_{i} \neq u_{j} \wedge \bigwedge_{\ell=1}^{d} E\left(u_{i_{\ell}}, u_{j_{\ell}}\right)\right),
$$

where $i$ and $j$ range over $[m]$ in the first conjunction, and $i_{1}, \ldots, i_{d}, j_{1}, \ldots, j_{d}$ are indices in $[m]$. The number $d$ of atomic facts in the conjunction is called the degree of $p$. The number $m$ of distinct variables is the order of $p$.

To the formula (3) we can associate the graph $H$ on the vertices $u_{1}, \ldots, u_{m}$ that has an edge between $u_{i_{\ell}}$ and $u_{j_{\ell}}$ for each $\ell \in[d]$, and the first $k$ vertices $u_{1}, \ldots, u_{k}$ distinguished from the rest. We use the 
notation $H\left(u_{1}, \ldots, u_{k}\right)$ for such kind of graphs, and $\oplus H\left(u_{1}, \ldots, u_{k}\right)$ to denote the FO[ $\oplus$ ]-polynomial corresponding to this graph. Note that the formula expresses the parity of the number of extensions of $u_{1}, \ldots, u_{k}$ to a copy of $H$. Note also that the degree of $\oplus H\left(u_{1}, \ldots, u_{k}\right)$ is the number of edges of $H$.

Example 1. If $H$ is a triangle containing vertex $u$, then $\oplus H(u)$ is the formula that expresses the parity of the number of extensions of $u$ to a triangle. Formally, $\oplus H(u)$ is the formula

$$
\oplus v \oplus w(u \neq v \wedge u \neq w \wedge v \neq w \wedge E(u, v) \wedge E(v, w) \wedge E(w, u)) .
$$

Note that over undirected graphs, this formula is always false. This is because for every triangle containing $u$, there are two assignments to the variables $v$ and $w$ which witness $H$. Thus, the total number of satisfying assignments is twice the number of triangles containing $u$ and is therefore always even. In general, if $H$ has an even number of automorphisms that fix $u_{1}, \ldots, u_{k}$, then $\oplus H\left(u_{1}, \ldots, u_{k}\right)$ will always be false, while for graphs with an odd number of automorphisms we get non-trivial formulas.

Remark 1. The observation at the end of Example 1 has one important consequence: if $H\left(u_{1}, \ldots, u_{k}\right)$ has $r$ isolated vertices outside $\left\{u_{1}, \ldots, u_{k}\right\}$ and $r \geq 2$, then $\oplus H\left(u_{1}, \ldots, u_{k}\right)$ is always false because then the number of automorphisms of $H$ that fix $u_{1}, \ldots, u_{k}$ is a multiple of $r$ !, which is even when $r \geq 2$. In particular, this means that in the general form of $\mathrm{FO}[\oplus]$-polynomials displayed in equation (3) we can always assume that

$$
m \leq 2 d+1 .
$$

This will be important because, by assuming it, lower bounds on the order imply lower bounds on the degree.

Remark 2. On graphs, an atomic formula of the form $E\left(u_{1}, u_{1}\right)$ is just false, while an atomic formula of the form $E\left(u_{1}, u_{2}\right)$ with distinct variables $u_{1}$ and $u_{2}$ is equivalent to $u_{1} \neq u_{2} \wedge E\left(u_{1}, u_{2}\right)$, which is an FO[ $\left.\oplus\right]-$ polynomial of order two and degree one. Similarly, an atomic formula of the form $u_{1}=u_{1}$ is just true, while an atomic formula of the form $u_{1}=u_{2}$ with distinct variables $u_{1}$ and $u_{2}$ is equivalent to the negation of the $\mathrm{FO}[\oplus]$-polynomial $u_{1} \neq u_{2}$; this has order two and degree zero. This means that every quantifier-free formula is equivalent to a Boolean combination of $\mathrm{FO}[\oplus]$-polynomials of order two and degree at most one.

Remark 3. There is a precise sense in which $\mathrm{FO}[\oplus]$-polynomials correspond to polynomials over the Boolean edge-variables $X_{u v}$. For example, the formula from Example 1 corresponds to the family of degree3 polynomials

$$
\sum_{\substack{v \in[n] \\ v \neq u}} \sum_{\substack{w \in[n] \\ w \neq u \\ w \neq v}} X_{u v} X_{v w} X_{w u} .
$$

as $u$ ranges over $[n]$.

\subsection{Independence and plan of action}

The formulas we are interested in are Boolean combinations of polynomials. Let $p(x, y, z)$ be such a formula and $Y(a, b, c)$ be the event that $p$ cannot distinguish any two permutations of $a, b, c$. The main obstacle to carrying out the argument sketched at the beginning of this section is that it is not true, in general, that the events $Y(a, b, c)$ and $Y\left(a^{\prime}, b^{\prime}, c^{\prime}\right)$ are almost independent, even if $a, b, c, a^{\prime}, b^{\prime}, c^{\prime}$ are all different. The 
reason is that the formula $p(x, y, z)$ may include statements about the graph $G$ which do not involve the free variables. These are true or false independently of the choice of $a, b, c$ or $a^{\prime}, b^{\prime}, c^{\prime}$ and thus create correlations between $Y(a, b, c)$ and $Y\left(a^{\prime}, b^{\prime}, c^{\prime}\right)$.

It is illustrative to give an example of this.

Example 2. Let $p(x, y)$ be the formula that is the conjunction of the following : (1) $\oplus z E(x, z)$ ( $x$ has odd degree), (2) $\neg \oplus z E(y, z)$ ( $y$ has even degree); and (3) $\oplus H$ for some fixed non-trivial rigid graph $H$ ( $x$ and $y$ do not appear free in this). This is a Boolean combination of $\mathrm{FO}[\oplus]$-polynomials of degree bounded by the number of edges of $H$.

Note that if $p(a, b)$ holds then $p(b, a)$ must fail. Therefore, the probability that $p(a, b) \nLeftarrow \neg p(b, a)$ holds is approximately $2 \cdot \frac{1}{8}$ since each of the three conditions in $p(a, b)$ holds with probability approximately $\frac{1}{2}$ almost independently, and similarly for $p(b, a)$. On the other hand, the probability that both $p(a, b) \nLeftarrow \rightarrow$ $p(b, a)$ and $p\left(a^{\prime}, b^{\prime}\right) \& p\left(b^{\prime}, a^{\prime}\right)$ hold simultaneously is approximately $4 \cdot \frac{1}{32}$. This is because in each of the four cases in which both hold, condition (3) either holds for both $a, b$ and $a^{\prime}, b^{\prime}$ or for neither (since $x$ and $y$ do not appear). We are left with five conditions that hold with probability approximately $\frac{1}{2}$ almost independently. Since $4 \cdot \frac{1}{32}$ is not $\epsilon$-close to $\left(2 \cdot \frac{1}{8}\right)^{2}$, this shows that $Y(a, b)$ and $Y\left(a^{\prime}, b^{\prime}\right)$ are not almost independent.

The example just sketched suggests that we factor out the condition that does not depend on neither $x$ nor $y$ from $p(x, y) \leftrightarrow p(y, x)$ since this is the cause for the statistical dependence between $Y(a, b)$ and $Y\left(a^{\prime}, b^{\prime}\right)$. However, while such an argument can be made to work in the example above, it is not clear what such a factoring would entail when $p$ contains disjunctions.

The key observation at this point is that the full type of $(x, y)$ in terms of its atomic type (the pattern of connections and equalities among $x$ and $y$ ) and the truth values of its $\oplus H$ 's as $H$ ranges over all small graphs that contain $x$ and $y$ as vertices is enough to determine the truth value of $p(x, y)$. Thus, if we were able to find a full type implying $p(x, y)$ that is symmetric in $x$ and $y$, we would have reduced the case of general $p(x, y)$ to the case of a $p(x, y)$ that consists of a single term and eliminated the need to consider disjunctions. The argument that we use is a bit more delicate than this, but this is the main idea.

\subsection{Normal forms}

In this section we introduce some definitions and discuss two different types of normal forms for Boolean combinations of $\mathrm{FO}[\oplus]$-polynomials.

An I-labeled graph is a graph with some vertices labeled by elements of $I$ in such a way that, for every $i \in I$ there is exactly one vertex labeled $i$, and the set of labeled vertices induces an independent set. The set of labeled vertices of an $I$-labeled graph $H$ is denoted by $\mathcal{L}(H)$. The vertex labeled by $i \in I$ is denoted by $H(i)$. An $I$-labeled graph $H$ is label-connected if $H \backslash \mathcal{L}(H)$ is connected. Let $\operatorname{Conn}_{I}^{t}$ be the set of all $I$-labeled label-connected graphs with at most $t$ unlabeled vertices. We say that $H$ depends on label $i \in I$ if $H(i)$ is not an isolated node. We say that $H$ is label-dependent if it depends on all its labels. Let Conn $I_{I}^{*, t}$ be the subset of all labeled graphs in $\mathrm{Conn}_{I}^{t}$ that are label-dependent.

A $k$-labeled graph is a $[k]$-labeled graph. A $\leq k$-labeled graph is an $I$-labeled graph for some $I \subseteq[k]$. Let $H$ be a $\leq k$-labeled graph with labels $I \subseteq[k]$. A homomorphism from $H$ to a pair $(G, \mathbf{a})$, where $G$ is a graph and $\mathbf{a}=\left(a_{1}, \ldots, a_{k}\right)$ is a tuple in $V(G)^{k}$, is a homomorphism $\chi \in \operatorname{Hom}(H, G)$ such that $\chi(H(i))=a_{i}$ for each $i \in I$. A homomorphism $\chi$ from $H$ to $(G, \mathbf{a})$ is injective if for any distinct $a, b \in V(H)$ such that $\{a, b\} \nsubseteq \mathcal{L}(H)$ we have $\chi(a) \neq \chi(b)$. Write $\oplus H(G, \mathbf{a})$ for the parity of the number of injective homomorphisms from $H$ to $(G, \mathbf{a})$. We usually omit $G$ and write $\oplus H(\mathbf{a})$. When $H$ is a $k$-labeled graph (i.e. $I=[k]$ ), the notation for this in [9] is $[H]_{2}(G, \mathbf{a})$. 
We call the number of vertices in $H$ the order of $H$ and the number of edges in $H$ the degree of $H$. These are the same as the order and degree respectively of the polynomial $\oplus H(\mathbf{x})$. Note that if $H$ has order $c$, then it has at most $c$ unlabeled vertices.

A $K K$-normal form of order $m$ with free-variables $\mathbf{x}=\left(x_{1}, \ldots, x_{k}\right)$ is a Boolean combination of the atomic types on the variables $\mathbf{x}$ and formulas $\oplus H(\mathbf{x})$ 's as $H$ ranges over the $k$-labeled label-connected graphs of order $m$ with labeled vertices $\mathbf{x}$. A regular normal form of order $m$ with free-variables $\mathbf{x}$ is a Boolean combination of the atomic types on the variables $\mathbf{x}$ and the $\oplus H(\mathbf{x})$ 's as $H$ ranges over the $\leq k$ labeled label-connected, label-dependent graphs of order $m$ with labeled vertices within $\mathbf{x}$.

Example 3. Let $\phi(x, y)$ be the formula

$$
\oplus z(E(x, z)) \wedge \neg \oplus z(E(y, z)),
$$

saying that $x$ has odd degree and $y$ has even degree. This is a regular normal form. On the other hand, it is not a KK-normal form because the formula $\oplus z(E(x, z))$ cannot be put in the form $\oplus H(x, y)$ for any 2labeled graph $H$. However, as we will see, it is not hard to transform $\phi(x, y)$ into an equivalent KK-normal form.

Example 4. Let $p(x, y)$ be the formula

$$
\left(x \neq y \wedge E(x, y) \wedge \neg \oplus H_{1}(x, y) \wedge \oplus H_{2}(x, y)\right) \vee\left(x \neq y \wedge \neg E(x, y) \wedge \oplus H_{1}(x, y) \wedge \neg \oplus H_{2}(x, y)\right),
$$

where $H_{1}$ is the 2-labeled label-connected graph that has three vertices $x, y$, and $z$ and a single edge between $x$ and $z$, and $H_{2}$ is the 2-labeled label-connected graph that has three vertices $x, y$, and $z$ and a single edge between $y$ and $z$. This is a KK-normal form. On the other hand, it is not a regular normal form because $H_{1}$ and $H_{2}$ are not label-dependent. However, as we will see, it is not hard to transform $\phi(x, y)$ into a regular normal form.

The two examples above are actually logically equivalent and it is a general fact that Boolean combinations of $\mathrm{FO}[\oplus]$-polynomials, KK-normal forms, and regular normal forms of the same order have the same expressive power.

Lemma 2. Let $k \geq 0$ and $m \geq k$ be integers and let $\phi\left(x_{1}, \ldots, x_{k}\right)$ be an $F O[\oplus]$-formula. The following are equivalent:

1. $\phi$ is equivalent to a Boolean combination of $F O[\oplus]$-polynomials of order at most $m$,

2. $\phi$ is equivalent to a KK-normal form of order at most $m$,

3. $\phi$ is equivalent to a regular normal form of order at most $m$.

Proof. We may assume that $m \geq 2$ since $\mathrm{FO}[\oplus]$-polynomials and normal forms of order one or less are trivial.

$(1) \Rightarrow(2)$ : We show how to transform an $\mathrm{FO}[\oplus]$-polynomial $p$ of order at most $m$ into an equivalent Boolean combination of atomic types and formulas of the form $\oplus H$ where $H$ is a $k$-labeled label-connected graph of order at most $m$. We do this in two steps: in the first step we do it with $k$-labeled graphs that are not necessarily label-connected, and in the second step we ensure the connectivity condition.

Let us say $p$ has the form

$$
p\left(y_{1}, \ldots, y_{t}\right)=\oplus y_{t+1} \cdots \oplus y_{s}\left(\bigwedge_{i \neq j} y_{i} \neq y_{j} \wedge \bigwedge_{\ell=1}^{d} E\left(y_{i_{\ell}}, y_{j_{\ell}}\right)\right)
$$


where the indices $i$ and $j$ range over $[s]$ in the first conjunct, $i_{\ell}$ and $j_{\ell}$ are also indices in $[s]$ for each $\ell \in[d]$, the free variables $y_{1}, \ldots, y_{t}$ are among $x_{1}, \ldots, x_{k}$, and the bound variables $y_{t+1}, \ldots, y_{s}$ are disjoint from $x_{1}, \ldots, x_{k}$. After renaming the variables, we may assume that $\left(y_{1}, \ldots, y_{t}\right)=\left(x_{1}, \ldots, x_{t}\right)$. Let $H$ be the $k$-labeled graph of order $s$ with labeled vertices $x_{1}, \ldots, x_{k}$, non-labeled vertices $y_{t+1}, \ldots, y_{s}$, and edges $\left\{y_{i_{\ell}}, y_{j_{\ell}}\right\}$ as $\ell$ ranges over $[d]$. We claim that for every graph $G$ and every $\mathbf{a}=\left(a_{1}, \ldots, a_{k}\right) \in V_{G}^{k}$, we have

$$
p(\mathbf{a}) \equiv \sum_{f \in \mathcal{F}} \sum_{\sigma \in \mathcal{S}^{f}} \sigma(\mathbf{a}) \cdot \oplus H^{f}(\mathbf{a}) \bmod 2,
$$

where

- $\mathcal{F}$ is the set of partial mappings $f:[s] \backslash[t] \rightarrow[k] \backslash[t]$ that are injective on their domain $\operatorname{Dom}(f)$, including the empty map,

- for every $f \in \mathcal{F}$, the set $\mathcal{S}^{f}$ consists of all atomic types on the variables $x_{1}, \ldots, x_{k}$ that contain the positive atomic type of each $y_{j}$ with $j \in \operatorname{Im}(f)$ over $x_{1}, \ldots, x_{t}$ in $H$, plus the inequalities $x_{i} \neq x_{j}$ for every pair $i, j \in[t]$ with $i \neq j$, every pair $i, j \in[k]$ with $i \in[t]$ and $j \in \operatorname{Im}(f)$, and every pair $i, j \in \operatorname{Im}(f)$ with $i \neq j$,

- for every $f \in \mathcal{F}$, the graph $H^{f}$ is the $k$-labeled graph that is obtained from $H$ by deleting all edges between some $y_{i}$ with $i \in \operatorname{Dom}(f)$ and $x_{1}, \ldots, x_{t}$, and by identifying $y_{i}$ with $x_{f(i)}$ for every $i \in$ $\operatorname{Dom}(f)$.

To see that (5) holds, note that the term $\sigma(\mathbf{a}) \cdot \oplus H^{f}(\mathbf{a})$ counts the parity of the number of injective homomorphisms from $H$ to $G$ that map $x_{i}$ to $a_{i}$ for every $i \in[t]$, and $y_{i}$ to $a_{f(i)}$ for every $i \in \operatorname{Dom}(f)$, subject to the condition that a satisfies the rest of atomic relations specified in $\sigma$. Since for each $f \in \mathcal{F}$ all allowed possibilities for the rest of atomic relations are included, the resulting count is precisely $p(\mathbf{a})$. Now note that $\mathcal{F}$ and $\mathcal{S}^{f}$ are finite for every $f \in \mathcal{F}$ and therefore the expression (5) is equivalent to a Boolean combination of atomic types and $\oplus H$ 's as required.

It remains to see how to ensure that the $H$ 's are label-connected while preserving the bound on the order. Conveniently, this was done in Lemma 5.6 from [9] and we refer the reader to it.

(2) $\Rightarrow(3)$ : We need to show how to transform a formula $\oplus H$ where $H$ is a $k$-labeled label-connected graph of order at most $m$ into an equivalent Boolean combination of quantifier-free formulas of the form $\oplus F$ where $F$ is a $\leq k$-labeled label-connected, label-dependent graph of order at most $m$. The transformation is done in two steps. In the first step we reduce the number of isolated labeled vertices in $H$ or the number of non-labeled vertices of $H$ at the expense of using possibly label-disconnected graphs. In the second step we get rid of the label-disconnected graphs. Indeed the second step is as in the proof of Lemma 5.6 in [9] so we need only take care of the first step.

If $H$ is already label-dependent, there is nothing to do. Otherwise let $x_{1}, \ldots, x_{k}$ be the set of labeled vertices of $H$, assume $x_{k}$ is isolated in $H$, and let $x_{k+1}, \ldots, x_{s}$ be the set of non-labeled vertices of $H$. Then, for every graph $G$ and for every $\mathbf{a}=\left(a_{1}, \ldots, a_{k}\right) \in V_{G}^{k}$, we claim that

$$
\oplus H(\mathbf{a}) \equiv \oplus H^{-x_{k}}(\mathbf{a})+\sum_{j \in[s] \backslash[k]} \sum_{\sigma \in \mathcal{S}^{j}} \sigma(\mathbf{a}) \cdot \oplus H^{x_{j}=x_{k}}(\mathbf{a}) \bmod 2,
$$

where:

- $H^{-x_{k}}$ is the $\leq k$-labeled graph that results from deleting $x_{k}$ in $H$, 
- $\mathcal{S}^{j}$ is the set of atomic types on the variables $x_{1}, \ldots, x_{k}$ that contain the positive atomic type of $x_{j}$ over $x_{1}, \ldots, x_{k-1}$ in $H$, plus the inequalities $x_{j} \neq x_{i}$ for every $i \in[k-1]$,

- $H^{x_{j}=x_{k}}$ is the $k$-labeled graph that results from deleting all edges from $x_{j}$ to a labeled vertex and identifying $x_{j}$ and $x_{k}$ in $H$.

To see why (6) holds note two facts. First, the sum over $j \in[s] \backslash[k]$ counts the parity of the number of injective homomorphisms from $H^{-x_{k}}$ into $(G, \mathbf{a})$ that have $a_{k}$ as the image of some non-labeled vertex. Second, since the term $\oplus H^{-x_{k}}(\mathbf{a})$ counts the parity of all injective homomorphisms from $H^{-x_{k}}$ into $(G, \mathbf{a})$, this means that each injective homomorphism from $H^{-x_{k}}$ into $(G, \mathbf{a})$ that has $a_{k}$ as the image of some nonlabeled vertex is counted exactly twice and cancels. What is left is the parity of the number of injective homomorphisms from $H^{-x_{k}}$ into $(G, \mathbf{a})$ that does not have $a_{k}$ as the image of some non-labeled vertex. This is precisely $\oplus H(\mathbf{a})$ because $x_{k}$ is isolated in $H$ and therefore the only constraint it puts on the injective homomorphisms from $H$ into $(G, \mathbf{a})$ is that non-labeled vertices are not mapped to $a_{k}$.

To conclude, note that $[s] \backslash[k]$ and $\mathcal{S}^{j}$ are finite for every $j \in[s] \backslash[k]$ and therefore (6) is a Boolean combination of atomic types and $\oplus H$ 's as required.

(3) $\Rightarrow(1)$ : First, each atomic type is a quantifier-free formula, and hence a Boolean combination of FO $[\oplus]$-polynomials of order at most two and degree at most one by Remark 2 . Here we use the assumption made at the beginning that $m \geq 2$.

Second, let $H$ be a $\leq k$-labeled graph of order at most $m$. Let $y_{1}, \ldots, y_{t}$ be its set of labeled vertices, which is a subset of $x_{1}, \ldots, x_{k}$, and let $y_{t+1}, \ldots, y_{s}$ be its set of unlabeled vertices, which is a set disjoint from $x_{1}, \ldots, x_{k}$. Then

$$
\oplus H(\mathbf{x}) \equiv \sum_{\sigma \in \mathcal{S}} \sigma(\mathbf{x}) \cdot \oplus H^{\sigma}(\mathbf{x}) \bmod 2
$$

where:

- $\mathcal{S}$ is the collection of all equality types on $y_{1}, \ldots, y_{t}$,

- $H^{\sigma}$ is the $\leq k$-labeled graph that results from $H$ by identifying every vertex $y_{j}$ with $j \in[t]$ with the vertex $y_{i}$ with smallest index $i \in[t]$ for which the equality $y_{i}=y_{j}$ appears in $\sigma$, and by deleting duplicated edges. If $i \neq j$ we say that $y_{i}$ survives and $y_{j}$ disappears. All vertices $y_{i}$ with $i \in[s] \backslash[t]$ survive.

For fixed $\sigma \in \mathcal{S}$, let $A$ be the set of pairs $(i, j) \in[s]^{2}$ such that both vertices $y_{i}$ and $y_{j}$ survive in $H^{\sigma}$. Finally, let $B$ be the subset of pairs $(i, j)$ in $A$ such that $\left\{y_{i}, y_{j}\right\}$ is an edge in $H^{\sigma}$. Then

$$
\sigma(\mathbf{x}) \cdot \oplus H^{\sigma}(\mathbf{x}) \equiv \oplus y_{t+1} \cdots \oplus y_{s}\left(\bigwedge_{(i, j) \in A} y_{i} \neq y_{j} \wedge \bigwedge_{(i, j) \in B} E\left(y_{i}, y_{j}\right)\right) .
$$

This is precisely an $\mathrm{FO}[\oplus]$-polynomial whose free variables are the $y_{i}$ with $i \in[t]$ that survive in $H^{\sigma}$. Its order is $s$, which is at most $m$. Since $\mathcal{S}$ is finite, this shows that (7) is a equivalent to a Boolean combination of $\mathrm{FO}[\oplus]$-polynomials of order at most $m$.

\subsection{Distribution of frequency vectors}

The frequency vector of order $t$ in a graph $G$ is the $\{0,1\}$-vector indexed by the set of all connected graphs with at most $t$ vertices where the component $H$ is $\oplus H(G)$, i.e. the parity of the number of occurrences of $H$ in $G$. Kolaitis and Kopparty [9] give an analysis of the distribution of frequency vectors in a random graph 
$G \sim G(n, 1 / 2)$, for constant $t$. Our aim in the present section is to extend this analysis to orders that grow with $n$ and to $\leq k$-labeled graphs.

Let $\mathrm{Conn}_{\leq k}^{t}$ be the set of all $\leq k$-labeled label-connected graphs with at most $t$ unlabeled vertices. Let $\mathrm{Conn}_{\leq k}^{*, t}$ be the subset of $\mathrm{Conn}_{\leq k}^{t}$ containing all graphs that are label-dependent. Let $G$ be a graph, let a be a tuple in $V(G)^{k}$, and let $t \geq 0$ be an integer. Let freq ${ }_{\leq k, G}^{*, t}(\mathbf{a})$ be the $\{0,1\}$-vector indexed by the elements $\operatorname{Conn}_{\leq k}^{*, t}$ that has $\oplus H(\mathbf{a})$ as its component indexed by $H$. Next we extend the definition of feasible frequency vectors from [9] to the setting of $\leq k$-labeled graphs. In defining $\operatorname{FFreq}^{*}(\tau, \leq k, t)$ we will restrict our attention to injective atomic types $\tau$. This simplifies matters significantly and is enough for our purposes. If $\tau$ is an injective atomic type on $x_{1}, \ldots, x_{k}$, let $\operatorname{FFreq}^{*}(\tau, \leq k, t)$ denote the set of all feasible frequency vectors. Explicitly, these are all the $\{0,1\}$-vectors indexed by $\operatorname{Conn}_{\triangleleft k}^{*, t}$ whose component $F$ belongs to $\operatorname{aut}(F) \cdot \mathbb{Z}_{2}$. Here aut $(F)$ denotes the number of automorphisms of $F$ that fix the labels. Let FFreq $_{n}^{*}(\tau, \leq k, t)$ denote the set of $f \in \operatorname{FFreq}^{*}(\tau, \leq k, t)$ such that $f_{K_{1}(\emptyset)}=n \bmod 2$, where $K_{1}(\emptyset)$ is the graph with no labels and exactly one unlabeled vertex.

The next lemma describes the distribution of $\operatorname{freq}_{<k, G}^{*, t}(\mathbf{a})$ in a random graph. This is analogous to Theorem 2.4 in [9], but see also the statement of Theorem 8.2 in [10] (about subgraph copies), on which Theorem 2.4 is based. Our statement deviates from theirs in two directions: in generalizing it from a constant number of vertices to a growing number of vertices up to $\log \log \log n$, and from $k$-labeled graphs to $\leq k$-labeled graphs.

Lemma 3. For every $k \geq 0$ there exists $n_{0} \geq 0$ such that for every $n \geq n_{0}$, every injective atomic type $\tau$ on $k$ variables, every $c \leq \log \log \log n$, and every $k$-tuple a of distinct elements in $[n]$, the distribution of freq $_{\leq k, G}^{*, c}(\mathbf{a})$ as $G \sim G(n, 1 / 2 \mid \tau(\mathbf{a}))$ is $2^{-\Omega_{k}(n / \log n)}$-close in statistical distance from the uniform distribution over $\operatorname{FFreq}_{n}^{*}(\tau, \leq k, c)$.

Here, the notation $\Omega_{k}$ refers to an unspecified multiplicative constant that depends only on $k$, and the notation $G(n, 1 / 2 \mid \tau(\mathbf{a}))$ denotes the uniform distribution over the graphs with vertex-set $[n]$ restricted to those on which the set of vertices in the tuple a induces the subgraph specified by the atomic type $\tau$.

Before we discuss its proof, it is worth pointing out the differences between the statement of Lemma 3 and the statements of Theorem 2.4 in [9] and the more general Theorem 8.2 in [10]. Our statement here extends them in two directions, and both of these require significant adaptation of the proof.

The first difference concerns the extension which takes us from a bounded number of vertices to a growing number of vertices up to $\log \log \log n$. In order to achieve this, we relax the statistical distance from $2^{-\Omega_{k}(n)}$ in [10] to $2^{-\Omega_{k}(n / \log n)}$ here. For our purposes, this weaker bound on the distance is not significant. The proof is obtained by adapting the one in [10]. For this we need to make an explicit calculation of an $\epsilon$-bound in Lemma 4.7 in [10]. It is conceivable that a more careful analysis would yield a better bound still. However, the bound we get is sufficient for our purposes.

The second difference concerns the extension from $k$-labeled graphs in Theorem 8.2 in [10] to $\leq k$ labeled graphs here. We need this extension to be able to execute the factoring argument sketched in Section 3.2. This modification in the statement introduces the additional restriction of label-dependency (the * in freq $\left.{ }^{*, t}\right)$. This is required since the result would not be true without it. Luckily, though, Lemma 2 tells us that we will be able to assume label-dependency without loss of generality. In this case discovering the right assumption is the essential step in the proof. Once the concept is defined, the proof again follows the original one. One final difference between our statement and Theorem 8.2 in [10] is that we are stating only the case $s=\ell^{\prime}=0$ of their theorem since any other case is irrelevant for our application. This also has the benefit of shortening the proof somewhat. 
Proof sketch of Lemma 3. Handling $\leq k$-labeled graphs instead of $k$-labeled graphs in the proof of Theorem 8.2 in [10] is not problematic until we realize that the sets of copies of $F$ and $F^{\prime}$ in $\left(\mathrm{K}_{n}, \mathbf{a}\right)$ need not be disjoint even if $F$ and $F^{\prime}$ are non-isomorphic; i.e. Proposition 8.1(2) from [10] fails if $I=[k]$ and $F$ and $F^{\prime}$ are $\leq k$-labeled graphs instead of $k$-labeled graphs. This happens, for example, if $F$ and $F^{\prime}$ are $\leq k$-labeled label-connected graphs that are identical except that $F^{\prime}$ has one more labeled vertex than $F$ that is isolated. On the other hand, if $F$ and $F^{\prime}$ are non-isomorphic and depend on all its labels, then it can be seen that the sets of copies are disjoint. This is enough to carry over the argument in [9].

However, in order to allow a growing $c$, we need the following lemma that makes the $\epsilon$-bound explicit in the conclusion of Lemma 4.7 in [10]. For the definition of the $\mu$-Gowers norm $\|g\|_{U^{d}, \mu}$ see Section 4.1 in [10].

Lemma 4. Let $g: \mathbb{Z}_{2}^{d} \rightarrow\{-1,1\}$ be given by $g(\mathbf{y})=(-1)^{\prod_{i=1}^{d} \mathbf{y}_{i}}$. Let $\mu$ be the uniform distribution on $\mathbb{Z}_{2}^{d}$. Then $\|g\|_{U^{d}, \mu}<1-\epsilon$ where $\epsilon>1-\exp \left(-2^{-d^{2}-2 d+1}\right)$.

Proof. Let $p_{0}=\mu^{(d)}\left(\mathbf{0}, \mathbf{t}_{0}\right)$ where $\mathbf{t}_{0}=(\mathbf{0}, \ldots, \mathbf{0})$ and let $p_{1}=\mu^{(d)}\left(\mathbf{0}, \mathbf{t}_{1}\right)$ where $\mathbf{t}_{1}=\left(\mathbf{e}_{1}, \ldots, \mathbf{e}_{d}\right)$ and $\mathbf{e}_{j}$ is the vector in $\mathbb{Z}_{2}^{d}$ that is 0 everywhere except at component $j$ where it is 1 . We claim that

$$
\left(\mathrm{D}_{\mathbf{t}_{0}} g\right)(\mathbf{0})=\prod_{S \subseteq[d]}(-1)^{\prod_{i=1}^{d}\left(\mathbf{0}_{i}+\sum_{j \in S} \mathbf{0}_{i}\right)}=\prod_{S \subseteq[d]}(-1)^{0}=1
$$

and

$$
\left(\mathrm{D}_{\mathbf{t}_{1}} g\right)(\mathbf{0})=\prod_{S \subseteq[d]}(-1)^{\prod_{i=1}^{d}\left(\mathbf{0}_{i}+\sum_{j \in S} \mathbf{e}_{j, i}\right)}=(-1)^{1} \cdot \prod_{S \subset[d]}(-1)^{0}=-1 .
$$

The first is clear. To see the second note that, whenever $S$ is a proper subset of $[d]$, the factor $\mathbf{0}_{i}+\sum_{j \in S} \mathbf{e}_{j, i}$ in the exponent vanishes at each $i \in[d] \backslash S$, and whenever $S=[d]$, the factor $\mathbf{0}_{i}+\sum_{j \in S} \mathbf{e}_{j, i}$ is 1 at each $i \in[d]$.

From the above,

$$
\begin{aligned}
\|g\|_{U^{d}, \mu}^{2^{d}} & =\left|\mathbb{E}_{(x, \mathbf{t}) \sim \mu^{(d)}}\left[\left(\mathrm{D}_{\mathbf{t}} g\right)(x)\right]\right|=\left|\sum_{(x, \mathbf{t})} \mu^{(d)}(x, \mathbf{t})\left(\mathrm{D}_{\mathbf{t}} g\right)(x)\right| \\
& \leq\left|p_{0} \cdot\left(\mathrm{D}_{\mathbf{t}_{0}} g\right)(\mathbf{0})+p_{1} \cdot\left(\mathrm{D}_{\mathbf{t}_{1}} g\right)(\mathbf{0})\right|+\sum_{\begin{array}{c}
(x, \mathbf{t}): \\
\left.(x)=\mathbf{t}_{0}\right) \\
(x, \mathbf{t}) \neq\left(\mathbf{0}, \mathbf{t}_{1}\right)
\end{array}} \mu^{(d)}(x, \mathbf{t}) \cdot\left|\left(\mathrm{D}_{\mathbf{t}} g\right)(x)\right| \\
& \leq\left|p_{0}-p_{1}\right|+1-p_{0}-p_{1},
\end{aligned}
$$

where the first inequality follows from the triangle inequality, and the second inequality follows from the computations above and the fact that each $\left(\mathrm{D}_{\mathbf{t}} g\right)(x)$ has unit magnitude.

Next we argue that if $\mu$ is the uniform distribution over $\mathbb{Z}_{2}^{d}$ then $\mu^{(i)}$ is the uniform distribution over $\left(\mathbb{Z}_{2}^{d}\right)^{i+1}$. We show, by induction on $i$, that $\mu^{(i)}\left(\mathbf{x}, \mathbf{t}_{1}, \ldots, \mathbf{t}_{i}\right)=2^{-d(i+1)}$ for every $\left(\mathbf{x}, \mathbf{t}_{1}, \ldots, \mathbf{t}_{i}\right) \in\left(\mathbb{Z}_{2}^{d}\right)^{i+1}$. Since $\mu^{(0)}=\mu$, the claim is clear for $i=0$. For $i>0$ we have

$$
\mu^{(i)}\left(\mathbf{x}, \mathbf{t}_{1}, \ldots, \mathbf{t}_{i}\right)=\frac{\mu^{(i-1)}\left(\mathbf{x}, \mathbf{t}_{1}, \ldots, \mathbf{t}_{i-1}\right) \cdot \mu^{(i-1)}\left(\mathbf{x}+\mathbf{t}_{i}, \mathbf{t}_{1}, \ldots, \mathbf{t}_{i-1}\right)}{\sum_{\mathbf{z}} \mu^{(i-1)}\left(\mathbf{z}, \mathbf{t}_{1}, \ldots, \mathbf{t}_{i-1}\right)} .
$$

Applying the induction hypothesis this is

$$
\frac{2^{-d i} \cdot 2^{-d i}}{2^{d} \cdot 2^{-d i}}=2^{-d(i+1)}
$$


Thus, $p_{0}=p_{1}=2^{-d(d+1)}$ and we get $\left|p_{0}-p_{1}\right|+1-p_{0}-p_{1}=1-2^{-d(d+1)+1}$. Putting it all together we conclude that

$$
\|g\|_{U^{d}, \mu} \leq\left(1-2^{-d(d+1)+1}\right)^{1 / 2^{d}} \leq e^{-2^{-d(d+1)+1} \cdot 2^{-d}}=e^{-2^{-d^{2}-2 d+1}}
$$

With the $\epsilon$-bound from Lemma 4 in hand we can mimic the proof of Theorem 8.2 in [10] to get the proof of Lemma 3. First we note that the bound in the conclusion of Theorem 4.8 in [10] for the special case in which $\mu$ is the uniform distribution is really $(1-\epsilon)^{r}$ for the $\epsilon$ from Lemma 4 . This translates into the same bound for the conclusions of Lemmas 4.1 and 3.3 in [10]. The bound in the conclusion of Lemma 3.3 in [10] is eventually plugged into the hypothesis of Vazirani's XOR Lemma, which returns the bound $2^{\ell} \cdot(1-\epsilon)^{r}$ on the statistical distance, where in our case $\ell$ is the number of $\leq k$-labeled label-connected label-dependent graphs with at most $c$ unlabeled vertices. A generous bound on this factor is $\ell \leq 2^{(c+k)^{2}+1}$. Now note that in the proof of Theorem 8.2 in [10], the remaining parameters are $r=\Omega_{k}(n / c)$ and $d=O\left(c^{2}\right)$. Using the bound on $\epsilon$ from Lemma 4, we get the following bound on the statistical distance:

$$
2^{\ell} \cdot(1-\epsilon)^{r} \leq 2^{(c+k)^{2}+1} \cdot\left(\exp \left(-2^{-O\left(c^{4}\right)}\right)\right)^{\Omega_{k}(n / c)} .
$$

For $c \leq \log \log \log n$ this is $2^{-\Omega_{k}(n / \log n)}$, as required.

\subsection{The argument itself}

Finally we are at the point where we can execute the plan sketched at the beginning of Section 3. Fix a positive integer $k$ (for the application in Section 5 it suffices to take $k=3$ ) and let $p\left(x_{1}, \ldots, x_{k}\right.$ ) be a regular normal form of order $c \leq \log \log \log n$. For every $\mathbf{a}=\left(a_{1}, \ldots, a_{k}\right) \in[n]^{k}$, define the following indicator random variables:

$$
\begin{aligned}
& X(\mathbf{a}):=\mathbb{I}\left[p(\mathbf{a}) \nLeftarrow \rightarrow p(\mathbf{a} \circ \pi) \text { for some } \pi \in S_{k}\right], \\
& Y(\mathbf{a}):=\mathbb{I}\left[p(\mathbf{a}) \leftrightarrow p(\mathbf{a} \circ \pi) \text { for every } \pi \in S_{k}\right] .
\end{aligned}
$$

Obviously, $X(\mathbf{a})=1-Y(\mathbf{a})$, and $Y(\mathbf{a})$ is the indicator random variable for the event that $p$ does not distinguish any two permuted versions of a. Our goal is to show that in a random graph $G$, with high probability, $Y(\mathbf{a})$ holds for some a, and for this we will follow the plan sketched at the beginning of this section.

Write $p(\mathbf{x})$ as a DNF on the (Boolean) variables

$$
\tau_{1}(\mathbf{x}), \ldots, \tau_{r}(\mathbf{x}), \oplus H_{1}(\mathbf{x}), \ldots, \oplus H_{\ell}(\mathbf{x}),
$$

where $\tau_{1}, \ldots, \tau_{r}$ are the atomic types on $x_{1}, \ldots, x_{k}$, and $H_{1}, \ldots, H_{\ell}$ are the $\leq k$-labeled label-connected, label-dependent graphs with labeled vertices within $x_{1}, \ldots, x_{k}$ and order at most $c$. Observe that, in particular, $H_{1}, \ldots, H_{\ell}$ have at most $c$ unlabeled vertices and thus belong to Conn $\mathrm{C}_{\leq k}^{*, c}$. Let us assume that $H_{1}, \ldots, H_{e}$ are the ones for which aut $\left(H_{i}\right)$ is odd, and that $H_{e+1}, \ldots, H_{\ell}$ are the rest. Also assume that $H_{1}, \ldots, H_{f}$ are the graphs from among $H_{1}, \ldots, H_{e}$ that do not have any label at all, and $H_{f+1}, \ldots, H_{e}$ are the rest. Since atomic types are mutually exclusive and each $\oplus H_{j}(\mathbf{a})$ is false for $j \in\{e+1, \ldots, \ell\}$, we may assume that each term in the DNF formula has the form

$$
\tau_{i}(\mathbf{x}) \cdot \prod_{j \in K} \oplus H_{j} \cdot \prod_{j \in K^{\prime}} \overline{\oplus H_{j}} \cdot \prod_{j \in I} \oplus H_{j}(\mathbf{x}) \cdot \prod_{j \in I^{\prime}} \overline{\oplus H_{j}(\mathbf{x})} .
$$


for some $i \in[r]$, some partition $\left(K, K^{\prime}\right)$ of $[f]$, and some partition $\left(I, I^{\prime}\right)$ of $[e] \backslash[f]$.

Next note that for every permutation $\pi \in S_{k}$, the sequence of Boolean variables $\tau_{1}(\mathbf{x} \circ \pi), \ldots, \tau_{r}(\mathbf{x} \circ$ $\pi)$ is equivalent to a permutation of the sequence of Boolean variables $\tau_{1}(\mathbf{x}), \ldots, \tau_{r}(\mathbf{x})$. Similarly, the sequence of Boolean variables $\oplus H_{f+1}(\mathbf{x} \circ \pi), \ldots, \oplus H_{e}(\mathbf{x} \circ \pi)$ is equivalent to a permutation of the sequence $\oplus H_{f+1}(\mathbf{x}), \ldots, \oplus H_{e}(\mathbf{x})$. Therefore $p(\mathbf{x})$ and $p(\mathbf{x} \circ \pi)$ are functions of the same Boolean variables and we can write $p(\mathbf{x} \circ \pi)$ also as a DNF formula with terms of the type (8).

From now on, for every $K \subseteq[f]$, let $R_{K}$ be the term

$$
R_{K}:=\prod_{j \in K} \oplus H_{j} \cdot \prod_{j \in K^{\prime}} \overline{\oplus H_{j}},
$$

where $K^{\prime}=[f] \backslash K$. Recall that $H_{1}, \ldots, H_{f}$ are all label-free and therefore $R_{K}$ does not depend on $\mathbf{x}$. Similarly, for every $I \subseteq[e] \backslash[f]$, let $S_{I}(\mathbf{x})$ be the term

$$
S_{I}(\mathbf{x}):=\prod_{j \in I} \oplus H_{j}(\mathbf{x}) \cdot \prod_{j \in I^{\prime}} \overline{\oplus H_{j}(\mathbf{x})},
$$

where $I^{\prime}=([e] \backslash[f]) \backslash I$. For every $K \subseteq[f]$, let $p_{K}(\mathbf{x})$ denote the disjunction of the terms in $p(\mathbf{x})$ that are consistent with $R_{K}$. Therefore $p(\mathbf{x})$ is equivalent to the disjunction $\bigvee_{K \subseteq[f]} p_{K}(\mathbf{x})$.

Define the "all-positive-term" as one in which $\oplus H_{j}(\mathbf{x})$ appears positively for all $j \in[e] \backslash[f]$ :

$$
Z_{K}(\mathbf{x}):=\sigma(\mathbf{x}) \cdot R_{K} \cdot S_{[e] \backslash[f]}(\mathbf{x}),
$$

where $\sigma$ is the atomic type that forces $x_{i} \neq x_{j}$ for $i \neq j$, and all possible edges among different $x_{i}, x_{j}$. We show that for every $\mathbf{a} \in[n]^{k}$, the event $Z_{K}(\mathbf{a})=1$ implies $p(\mathbf{a}) \leftrightarrow p(\mathbf{a} \circ \pi)$ for every $\pi \in S_{k}$.

Lemma 5. $Z_{K}(\mathbf{a}) \leq Y(\mathbf{a})$.

Proof. Fix a permutation $\pi \in S_{k}$. First note that the choice of $\sigma$ guarantees that $\sigma(\mathbf{a})$ is equivalent to $\sigma(\mathbf{a} \circ \pi)$. Also, the sequence $\oplus H_{f+1}(\mathbf{a} \circ \pi), \ldots, \oplus H_{e}(\mathbf{a} \circ \pi)$ is equivalent to a permutation of the sequence $\oplus H_{f+1}(\mathbf{a}), \ldots, \oplus H_{e}(\mathbf{a})$, and all appear positively in $Z_{K}(\mathbf{a})$. It follows from these facts that either the term $Z_{K}(\mathbf{a})$ appears in both DNFs for $p(\mathbf{a})$ and $p(\mathbf{a} \circ \pi)$, or in neither. If it appears in both, then clearly $Z_{K}(\mathbf{a})=1$ implies both $p(\mathbf{a})$ and $p(\mathbf{a} \circ \pi)$. If it does not appear in either, then $Z_{K}(\mathbf{a})=1$ implies $\overline{p(\mathbf{a})}$ and $\overline{p(\mathbf{a} \circ \pi)}$ since $Z_{K}(\mathbf{a})$ is incompatible with any other term of the DNFs for $p(\mathbf{a})$ and $p(\mathbf{a} \circ \pi)$. In either case, $Z_{K}(\mathbf{a})$ implies $p(\mathbf{a}) \leftrightarrow p(\mathbf{a} \circ \pi)$.

At this point it will suffice to show that for every $K \subseteq[f]$, the event $Z_{K}(\mathbf{a})=1$ holds for some $\mathbf{a} \in[n]^{k}$ with high probability in the probability space conditioned on $R_{K}$. From now on, for every event $A$, write

$$
\mathbb{P}_{K}[A]:=\mathbb{P}\left[A \mid R_{K}\right] .
$$

Let us start by computing the probability of $Z_{K}(\mathbf{a})$ for $\mathbf{a} \in[n]^{k}$ with $a_{i} \neq a_{j}$ for $i \neq j$ in this probability space. Let $\delta$ be the maximum, over all injective atomic types $\tau(\mathbf{x})$, of the statistical distance between the distribution freq ${ }_{\leq k, G}^{*, c}(\mathbf{a})$ as $G \sim G(n, 1 / 2 \mid \tau(\mathbf{a}))$ and the uniform distribution over $\operatorname{FFreq}^{*}(\tau, \leq k, c)$. Note that, by symmetry, $\delta$ does not depend on a provided $a_{i} \neq a_{j}$ for $i \neq j$.

\section{Lemma 6.}

$$
\mathbb{P}_{K}\left[Z_{K}(\mathbf{a})\right] \leq\left(\frac{2^{-e}}{2^{-f}}+\delta \cdot \frac{1}{2^{-f}-\delta}+\delta \cdot \frac{2^{-e}}{2^{-f} \cdot\left(2^{-f}-\delta\right)}\right) \cdot 2^{-\left(\begin{array}{c}
k \\
2
\end{array}\right)}
$$

and

$$
\mathbb{P}_{K}\left[Z_{K}(\mathbf{a})\right] \geq\left(\frac{2^{-e}}{2^{-f}}-\delta \cdot \frac{1}{2^{-f}+\delta}-\delta \cdot \frac{2^{-e}}{2^{-f} \cdot\left(2^{-f}+\delta\right)}\right) \cdot 2^{-\left(\begin{array}{c}
k \\
2
\end{array}\right)} .
$$


Proof. We have

$$
\mathbb{P}_{K}\left[Z_{K}(\mathbf{a})\right]=\frac{\mathbb{P}\left[Z_{K}(\mathbf{a}) \cdot R_{K}\right]}{\mathbb{P}\left[R_{K}\right]}=\frac{\mathbb{P}\left[S_{[e] \backslash[f]}(\mathbf{a}) \cdot R_{K} \mid \sigma(\mathbf{a})\right] \cdot \mathbb{P}[\sigma(\mathbf{a})]}{\mathbb{P}\left[R_{K}\right]}
$$

The denominator is at most $2^{-f}+\delta$ and at least $2^{-f}-\delta$ by choice of $\delta$. Similarly, the numerator is at most

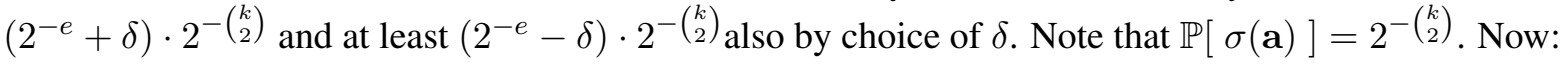

$$
\frac{2^{-e}+\delta}{2^{-f}-\delta}-\frac{2^{-e}}{2^{-f}}=\frac{2^{-e} \cdot 2^{-f}+\delta \cdot 2^{-f}-2^{-e} \cdot 2^{-f}+2^{-e} \cdot \delta}{2^{-f} \cdot\left(2^{-f}-\delta\right)}
$$

which simplifies to

$$
\delta \cdot \frac{1}{2^{-f}-\delta}+\delta \cdot \frac{2^{-e}}{2^{-f} \cdot\left(2^{-f}-\delta\right)}
$$

Similarly,

$$
\frac{2^{-e}}{2^{-f}}-\frac{2^{-e}-\delta}{2^{-f}+\delta}=\delta \cdot \frac{1}{2^{-f}+\delta}+\delta \cdot \frac{2^{-e}}{2^{-f} \cdot\left(2^{-f}+\delta\right)}
$$

Next we compute, for every $\mathbf{a}, \mathbf{a}^{\prime} \in[n]^{k}$ with all $a_{1}, \ldots, a_{k}, a_{1}^{\prime}, \ldots, a_{k}^{\prime}$ distinct, the probability of $Z_{K}(\mathbf{a}) \cdot Z_{K}\left(\mathbf{a}^{\prime}\right)$ in the probability space conditioned on $R_{K}$. Let $\gamma$ be the maximum, over all injective atomic types $\tau\left(\mathbf{x}, \mathbf{x}^{\prime}\right)$, of the statistical distance between the distribution $\operatorname{freq}_{\leq 2 k, G}^{*, c}\left(\mathbf{a}, \mathbf{a}^{\prime}\right)$ as $G \sim$ $G\left(n, 1 / 2 \mid \tau\left(\mathbf{a}, \mathbf{a}^{\prime}\right)\right)$ and the uniform distribution over $\operatorname{FFreq}^{*}(\tau, \leq 2 k, c)$. Note that, by symmetry, $\gamma$ does not depend on $\mathbf{a}, \mathbf{a}^{\prime}$ provided they are all distinct.

\section{Lemma 7.}

$$
\mathbb{P}_{K}\left[Z_{K}(\mathbf{a}) \cdot Z_{K}\left(\mathbf{a}^{\prime}\right)\right] \leq\left(\frac{2^{-2 e}}{2^{-2 f}}+\gamma \cdot \frac{1}{2^{-f}-\gamma}+\gamma \cdot \frac{2^{-2 e+f}}{2^{-f} \cdot\left(2^{-f}-\gamma\right)}\right) \cdot 2^{-2\left(\begin{array}{c}
k \\
2
\end{array}\right)} .
$$

Proof. Let $A$ denote the event that $\sigma(\mathbf{a})$ and $\sigma\left(\mathbf{a}^{\prime}\right)$ both hold. We have

$$
\begin{aligned}
\mathbb{P}_{K}\left[Z_{K}(\mathbf{a}) \cdot Z_{K}\left(\mathbf{a}^{\prime}\right)\right] & =\frac{\mathbb{P}\left[Z_{K}(\mathbf{a}) \cdot Z_{K}\left(\mathbf{a}^{\prime}\right) \cdot R_{K}\right]}{\mathbb{P}\left[R_{K}\right]} \\
& =\frac{\mathbb{P}\left[S_{[e] \backslash[f]}(\mathbf{a}) \cdot S_{[e] \backslash[f]}\left(\mathbf{a}^{\prime}\right) \cdot R_{K} \mid A\right] \cdot \mathbb{P}[A]}{\mathbb{P}\left[R_{K}\right]} .
\end{aligned}
$$

The denominator is at least $2^{-f}-\gamma$ by choice of $\gamma$. The numerator is at most $\left(2^{-2 e+f}+\gamma\right) \cdot 2^{-2\left(\begin{array}{c}k \\ 2\end{array}\right)}$ also by choice of $\gamma$. The trailing $2^{-2\left(\begin{array}{c}k \\ 2\end{array}\right)}$ factor is $\mathbb{P}[A]$. Now:

$$
\frac{2^{-2 e+f}+\gamma}{2^{-f}-\gamma}-\frac{2^{-2 e+f}}{2^{-f}}=\frac{2^{-2 e+f} \cdot 2^{-f}+\gamma \cdot 2^{-f}-2^{-2 e+f} \cdot 2^{-f}+2^{-2 e+f} \cdot \gamma}{2^{-f} \cdot\left(2^{-f}-\gamma\right)}
$$

which simplifies to

$$
\gamma \cdot \frac{1}{2^{-f}-\gamma}+\gamma \cdot \frac{2^{-2 e+f}}{2^{-f} \cdot\left(2^{-f}-\gamma\right)}
$$


Let us note at this point that the number of $\leq k$-labeled graphs of order at most $c$ is bounded by $2^{c^{2}+1}$. Therefore, using the bound $c \leq \log \log \log n$, we have $\ell \leq \frac{1}{2} \log n$ for sufficiently large $n$, and in particular

$$
2^{\ell} \leq \sqrt{n}
$$

We use this to prove the main consequence of this analysis up to now:

Lemma 8. Let $\mathbf{a}, \mathbf{a}^{\prime} \in[n]^{k}$ be such that $a_{1}, \ldots, a_{k}, a_{1}^{\prime}, \ldots, a_{k}^{\prime}$ are all different. The following hold:

1. $\mathbb{P}_{K}\left[Z_{K}(\mathbf{a})\right] \geq n^{-1 / 2} \cdot 2^{-\left(\begin{array}{c}k \\ 2\end{array}\right)}-2^{-\Omega_{k}(n / \log n)}$.

2. $\left|\mathbb{P}_{K}\left[Z_{K}(\mathbf{a}) \cdot Z_{K}\left(\mathbf{a}^{\prime}\right)\right]-\mathbb{P}_{K}\left[Z_{K}(\mathbf{a})\right] \cdot \mathbb{P}_{K}\left[Z_{K}\left(\mathbf{a}^{\prime}\right)\right]\right| \leq 2^{-\Omega_{k}(n / \log n)}$.

Proof. By Lemma 3, both $\delta$ and $\gamma$ are $2^{-\Omega_{k}(n / \log n)}$. On the other hand we have $2^{e-f} \leq 2^{e} \leq 2^{\ell} \leq n^{1 / 2}$ by (9) and also $2^{f} \leq 2^{\ell} \leq n^{1 / 2}$ by (9). Therefore $2^{f-e} \geq 2^{-e} \geq 2^{-\ell} \geq n^{-1 / 2}$ and $2^{-f} \geq 2^{-\ell} \geq n^{-1 / 2}$. Now 1 follows from plugging these bounds into the lower bound in Lemma 6 and 2 follows from plugging these bounds into the upper bound in Lemma 6 and the bound in Lemma 7 and recalling that $k$ is a constant.

Now we conclude by proving the main result of this section:

Lemma 9. For every $k>0$ and $\epsilon>0$, there exists $n_{0} \geq 0$ such that for every $n \geq n_{0}$ and every regular form $p\left(x_{1}, \ldots, x_{k}\right)$ of order bounded by $\log \log \log n$, for $G \sim G(n, 1 / 2)$, the probability that there exists $\mathbf{a} \in[n]^{k}$ with $a_{i} \neq a_{j}$ for $i \neq j$ such that $p(\mathbf{a}) \leftrightarrow p(\mathbf{a} \circ \pi)$ for every $\pi \in S_{k}$ is at least $1-\epsilon$.

Proof. Fix $k$ and $\epsilon$ and choose $n_{0}$ large. Let $m=\lfloor n / k\rfloor$. Divide $[n]$ into $m$ disjoint $k$-tuples $\left(\mathbf{a}_{1}, \ldots, \mathbf{a}_{m}\right)$ arbitrarily but in such a way that $a_{\ell, i} \neq a_{\ell, j}$ for $i \neq j$. Define: $Y=\sum_{\ell \in[m]} Y\left(\mathbf{a}_{\ell}\right)$ and $Z_{K}=\sum_{\ell \in[m]} Z_{K}\left(\mathbf{a}_{\ell}\right)$, the second for every $K \subseteq[f]$. Note that by Lemma 5 we have $Z_{K} \leq Y$. We want to show that $\mathbb{P}_{K}\left[Z_{K}=0\right] \leq \epsilon$. This will be enough since then

$$
\mathbb{P}[Y=0]=\sum_{K \subseteq[e]} \mathbb{P}\left[Y=0 \mid R_{K}\right] \cdot \mathbb{P}\left[R_{K}\right] \leq \sum_{K \subseteq[e]} \mathbb{P}_{K}\left[Z_{K}=0\right] \cdot \mathbb{P}\left[R_{K}\right] \leq \epsilon .
$$

To show that $\mathbb{P}_{K}\left[Z_{K}=0\right] \leq \epsilon$ we proceed by the second moment method. To simplify notation, let us fix $K \subseteq[f]$ and abbreviate $Z_{K}$ by $Z$, and $Z_{K}\left(\mathbf{a}_{\ell}\right)$ by $Z_{\ell}$. Similarly, all expectations $\mathbb{E}$, variances $\mathbb{V}$, and probabilities $\mathbb{P}$ appearing below refer to the probability space $\mathbb{P}_{K}$. In computing the variance $\mathbb{V}[Z]=$ $\mathbb{E}\left[Z^{2}\right]-\mathbb{E}[Z]^{2}$ we have

$$
\begin{aligned}
\mathbb{E}\left[Z^{2}\right]-\mathbb{E}[Z]^{2} & =\sum_{i, j} \mathbb{E}\left[Z_{i} \cdot Z_{j}\right]-\sum_{i, j} \mathbb{E}\left[Z_{i}\right] \cdot \mathbb{E}\left[Z_{j}\right] \\
& \leq \sum_{i} \mathbb{E}\left[Z_{i}^{2}\right]+2 \cdot \sum_{i \neq j}\left(\mathbb{E}\left[Z_{i} \cdot Z_{j}\right]-\mathbb{E}\left[Z_{i}\right] \cdot \mathbb{E}\left[Z_{j}\right]\right) \\
& \leq \sum_{i} \mathbb{E}\left[Z_{i}\right]+2 \cdot \sum_{i \neq j}\left(\mathbb{P}\left[Z_{i} \cdot Z_{j}\right]-\mathbb{P}\left[Z_{i}\right] \cdot \mathbb{P}\left[Z_{j}\right]\right) \\
& =\mathbb{E}[Z]+2 \cdot\left(\begin{array}{c}
m \\
2
\end{array}\right) \cdot 2^{-\Omega_{k}(n / \log n)} \\
& =\mathbb{E}[Z]+2^{-\Omega_{k}(n / \log n)},
\end{aligned}
$$

where the first inequality follows from considering the case $i=j$ in the first double sum and ignoring it in the second, the next inequality follows from the fact that $Z_{i}$ is a $0-1$-random variable, the equality after 
it follows from Lemma 8.2 (recall that $\mathbb{P}$ really stands for $\mathbb{P}_{K}$ here), and the last equality follows from $m=\lfloor n / k\rfloor$ and the fact that $k$ is a constant.

Now by Lemma 8.1 we have

$$
\mathbb{E}[Z] \geq m \cdot\left(n^{-1 / 2} \cdot 2^{-\left(\begin{array}{c}
k \\
2
\end{array}\right)}-2^{-\Omega_{k}(n / \log n)}\right)=\Omega_{k}\left(n^{1 / 2}\right) .
$$

Applying it to Chebyshev's inequality we obtain

$$
\mathbb{P}[Z=0] \leq \frac{\mathbb{V}[Z]}{\mathbb{E}[Z]^{2}} \leq \frac{\mathbb{E}[Z]+2^{-\Omega_{k}(n / \log n)}}{\mathbb{E}[Z]^{2}} \leq \epsilon
$$

for sufficiently large $n$.

\section{Defining a linear pre-order of width two}

In this section we construct the formula of very low depth that defines a linear pre-order of width 2 with high probability. The proof strategy is to analyse a variant of an algorithm for graph canonization due to Karp [8], and to exploit its massive implicit parallelism to get formulas of very low depth.

\subsection{Plan of action}

Informally, the graph canonization algorithm works as follows. For a given graph $G$, split the vertices into two classes: those of even degree and those of odd degree. Inductively, we split the classes further by dividing the vertices according to the parity of the numbers of neighbours they have in each of the existing classes. We continue this process until no more classes are split.

We will need three facts about this process: (1) that for $G \sim G(n, 1 / 2)$ the process will reach a state where each class has at most two vertices with high probability, (2) that this will happen in fewer than $n$ "generations" of the splitting process with high probability, and (3) that the process is massively parallel: all the classes created between the $\ell / 2$-th generation and the $\ell$-th generation are definable in terms of the classes created in the $\left(\log _{2} \ell\right)$-th generation.

\subsection{Splitting procedure}

Let $G=(V, E)$ be an undirected graph. For a vertex $x$ and a set $B$, we write $p(G, x, B)$ for the parity of the number of neighbours that $x$ has in $B$. We extend this to sets:

$$
p(G, A, B)=\sum_{x \in A} p(G, x, B) \bmod 2 .
$$

A splitting tree for $G$ is a rooted binary tree $T$ with each node $t$ carrying a label $L_{t} \subseteq V$ and a sign $M_{t} \in\{+,-\}$ denoting whether $t$ is marked or unmarked, and satisfying the following properties:

1. the label of the root is $V$,

2. no two siblings are marked,

3. if $t$ is an internal node, then ${ }^{1} L_{t 0} \cup L_{t 1}=L_{t}$ and $L_{t 0} \cap L_{t 1}=\emptyset$,

\footnotetext{
${ }^{1}$ Karp requires also $L_{t 0} \neq \emptyset$ and $L_{t 1} \neq \emptyset$. For us it is convenient to not require it and Karp's analysis still goes through with minor modifications that we point out.
} 
4. if $s$ is a leaf, $x, y \in L_{s}$ and $t$ is marked, then $p\left(G, x, L_{t}\right)=p\left(G, y, L_{t}\right)$.

Given a splitting tree $T$ for $G$, let $R(T)$ denote the set of unmarked nodes that are either the root or are a left child. Let $R^{\prime}(T)$ be the subset of $R(T)$ containing the root and all nodes $t$ such that the label of $t$ and its sibling are both non-empty ${ }^{2}$. One step of the splitting procedure works as follows:

1. let $t$ be the least node in $R(T)$ in level-order ${ }^{3}$ and mark it,

2. for every leaf $s$, let $L_{s a}:=\left\{x \in L_{s}: p\left(G, x, L_{t}\right)=a\right\}$ for both $a=0$ and $a=1$,

3. make ${ }^{4} s 0$ and $s 1$ the left and right children of $s$ and leave them unmarked.

Let $\mathcal{P}(T)$ be the result of applying one step of the splitting procedure to $T$. If the node $t$ that is chosen in the first step also belongs to $R^{\prime}(T)$ we say that the step is proper, otherwise improper. When $R^{\prime}(T)$ is empty we say that the procedure stalls at $T$. Note that when it stalls it will never make a proper step again. The procedure starts at the splitting tree $T_{0}$ that has only an unmarked root labeled by $V$.

\subsection{Analysis of the splitting procedure}

Let $T_{0}$ be the tree that has only an unmarked root labeled by $V$. For $k \geq 1$, let $T_{k}:=\mathcal{P}\left(T_{k-1}\right)$. Ideally we would like to show that after a modest number of steps, all leaves of the splitting tree are labeled by singletons or empty sets. Unfortunately the splitting procedure is not able to produce a tree with this property in general, not even with high probability on a random graph. The best we will be able to show is that for a randomly generated graph, with high probability all leaves will have at most two vertices.

We identify three key desirable properties of $T_{k}$, where the third is our goal:

$\left(A_{k}\right): T_{k}$ has $L_{t} \neq \emptyset$ for every node $t$,

$\left(B_{k}\right): T_{k}$ has been generated through proper steps only,

$\left(C_{k}\right): T_{k}$ has $\left|L_{t}\right| \leq 2$ for every leaf $t$.

In the following we will show:

1. property $\left(A_{k}\right)$ holds with high probability for suitable values of $k$,

2. property $\left(A_{k}\right)$ implies $\left(B_{2^{k}}\right)$ for every graph and every $k \geq 0$,

3. conditioned on $\left(B_{2^{k}}\right)$, property $\left(C_{2^{k}}\right)$ holds with high probability for suitable values of $k$.

Before we analyse the probability of $\left(A_{k}\right)$ we need to introduce some terminology and a lemma from [8]. Let $T$ be a splitting tree for some graph $H$ on the vertices $V$. To every node $t \in R^{\prime}(T)$ we associate a set $S_{t} \subseteq V$ : the set of all $x$ for which $t$ is the maximal node in $R^{\prime}(T)$ such that $L_{t}$ contains $x$. Let $\mathcal{S}(T)$ be the collection of all such sets. For every $t \in R^{\prime}(T)$, let $\beta_{t}$ be $t$ together with the set of nodes $s \in R^{\prime}(T)$ such that $s \neq t$ and $L_{s}$ is a maximal subset of $L_{t}$. Note that $S_{t}=\triangle_{s \in \beta_{t}} L_{s}$, where $\triangle$ denotes symmetric difference. Define $\ell\left(x, S_{t}\right):=\sum_{s \in \beta_{t}} p\left(H, x, L_{s}\right) \bmod 2$. We will say that another graph $G$ on the vertices $V$ is consistent with $T$ if $p\left(G, x, L_{t}\right)=p\left(H, x, L_{t}\right)$ holds for every $x \in V$ and every node $t \in R^{\prime}(T)$.

We state a consequence of Lemmas 4 and 5 in $[8]^{5}$ :

\footnotetext{
${ }^{2}$ Karp defines $R(T)$ as the set of unmarked nodes $t$ that are either the root or that have a sibling $t^{\prime}$ such that $\left|L_{t^{\prime}}\right|>\left|L_{t}\right|$, or $\left|L_{t^{\prime}}\right|=\left|L_{t}\right|$ and are a left child. This difference is inessential to the analysis. The only important point is to unambiguously choose one of the two children when both are unmarked and non-empty.

${ }^{3}$ Karp used symmetric order. This difference is not essential for Karp's analysis but is important for us.

${ }^{4}$ Karp's version makes this step only if $L_{s a} \neq \emptyset$ for both $a=0$ and $a=1$; this note is related to footnote 1 .

${ }^{5}$ It would seem from Lemma 4 in [8] that we also need the constraint $p\left(G, L_{t}, L_{t}\right)=0$. However, in our notation this constraint is implicit since $p\left(G, L_{t}, L_{t}\right)$ counts each edge within $L_{t}$ exactly twice.
} 
Lemma 10. Let $T$ be the splitting tree of some graph on the vertices $V$ and let $H$ be chosen uniformly at random among the graphs on the vertices $V$ that are consistent with $T$. If $t$ is a node in $R^{\prime}(T)$, then the distribution of $\left\{p\left(H, x, L_{t}\right)\right\}_{x \in V}$ is uniform over the assignments that satisfy the constraints

$$
p\left(H, S, L_{t}\right)=\ell\left(L_{t}, S\right) \text { for every } S \in \mathcal{S}\left(T_{k}\right) \backslash\{Y\},
$$

where $Y$ is the unique set in $\mathcal{S}(T)$ of which $L_{t}$ is a proper subset.

In order to be able to make use of this lemma it is important to notice that if $G$ denotes a random graph drawn from $G(n, 1 / 2)$ and $T_{0}, T_{1}, \ldots$ denotes the random sequence of splitting trees produced by this random graph, then the distribution of $T_{k+1}$ conditioned on $T_{k}$ is equally produced as follows: first choose a graph $H$ uniformly at random among those consistent with $T_{k}$, and then run one step of the splitting procedure on $T_{k}$ with respect to $H$. This follows from the fact that the restriction of a uniform distribution to a subset of its support is uniformly distributed on that subset.

Now we can analyse the probability of $\left(A_{k}\right)$ :

Lemma 11. Let $n \geq 1$ and $k \geq 1$ be integers such that $4 k \leq \log _{2} n$, and let $G \sim G(n, 1 / 2)$. Then, the probability that $\left(A_{k}\right)$ fails is $2^{k+1} \cdot \exp \left(-n / 2^{6 k}\right)$.

Proof. In order to simplify notation, in this proof we let $n_{t}:=\left|L_{t}\right|$. For a node $t$ at depth $\ell \leq k$ in $T_{k}$, we say that $t$ is unbiased if $\left|n_{t}-n \cdot 2^{-\ell}\right| \leq n \cdot 2^{-(2 k-\ell+1)}$ holds, and biased otherwise. Note for later use that we allow the error-term $n \cdot 2^{-(2 k-\ell+1)}$ to grow with $\ell$, but that it always stays below $n \cdot 2^{-\ell}$ because $\ell \leq k$. Let us consider the event defined as follows:

$\left(A_{k}^{\prime}\right): T_{k}$ has every node unbiased.

Note that since the error-term for $\ell=k$ is smaller than $n \cdot 2^{-k}$, property $\left(A_{k}^{\prime}\right)$ implies $\left(A_{k}\right)$. Thus, it suffices to bound the probability that $\left(A_{k}^{\prime}\right)$ fails.

Since $\left(A_{0}^{\prime}\right)$ holds, if $\left(A_{k}^{\prime}\right)$ fails then there is a largest $\ell \in\{0, \ldots, k-1\}$ such that $\left(A_{\ell}^{\prime}\right)$ is true and $\left(A_{\ell+1}^{\prime}\right)$ is false. Fix $\ell \in\{0, \ldots, k-1\}$, a leaf $r$ of $T_{\ell}$, and $a \in\{0,1\}$, and we bound the probability that the child $r a$ of $r$ becomes biased in $T_{\ell+1}$ conditioned on $T_{\ell}$ satisfying $\left(A_{\ell}^{\prime}\right)$. Let $t$ be the node with respect to which the splitting step $\ell+1$ is made. Since we are assuming that $T_{\ell}$ satisfies $\left(A_{\ell}^{\prime}\right)$, each label is non-empty and therefore $t$ belongs to $R^{\prime}\left(T_{\ell}\right)$. Let $Y$ be the unique set in $\mathcal{S}\left(T_{\ell}\right)$ of which $L_{t}$ is a proper subset. By the discussion after Lemma 10 , the tree $T_{\ell+1}$ can be seen as produced by first choosing $H$ uniformly at random among the graphs that are consistent with $T_{\ell}$, and then applying the splitting procedure on $T_{\ell}$ with respect to $t$ and $H$. By Lemma 10, the distribution of $\left\{p\left(H, x, L_{t}\right)\right\}_{x \in V}$ is uniform over the assignments that satisfy the constraints

$$
p\left(H, S, L_{t}\right)=\ell\left(L_{t}, S\right)
$$

for every $S$ in $\mathcal{S}\left(T_{\ell}\right) \backslash\{Y\}$. In particular, since all sets in $\mathcal{S}\left(T_{\ell}\right)$ are pairwise disjoint, if $S$ is the unique minimal set in $\mathcal{S}\left(T_{\ell}\right)$ that contains $L_{r}$, then the distribution of $\left\{p\left(H, x, L_{t}\right)\right\}_{x \in S}$ is uniform over the assignments that satisfy the constraint (10) for this $S$ only, or no constraint at all if $S=Y$.

Since $r$ is unbiased, the set $L_{r}$ is non-empty. Fix $x_{0} \in L_{r} \subseteq S$. A different way of generating the distribution $\left\{p\left(H, x, L_{t}\right)\right\}_{x \in S}$ without sampling $H$ is by first choosing values for $p\left(-, x, L_{t}\right)$ for $x \in$ $S \backslash\left\{x_{0}\right\}$ uniformly and independently at random, and then setting the value for $p\left(-, x_{0}, L_{t}\right)$ to the unique value that satisfies the constraint $p\left(-, S, L_{t}\right)=\ell\left(L_{t}, S\right)$, or setting it uniformly and independently at random if $S=Y$. In either case, the number $X$ of elements $x$ in $L_{r} \backslash\left\{x_{0}\right\}$ for which $p\left(-, x, L_{t}\right)=a$ is a random variable distributed according to the binomial distribution $\mathrm{B}\left(m, \frac{1}{2}\right)$ with $m=n_{r}-1$. Note for later use that 
$\left|n_{r a}-X\right| \leq 1$ because only $x_{0}$ could be missed in the count. By Hoeffding's inequality for the binomial distribution, the probability that $\left|X-\frac{1}{2} \cdot m\right| \geq t$ is bounded by $2 e^{-2 t^{2} / m}$, which is bounded by

$$
2 e^{-2 t^{2} /\left(2 n \cdot 2^{-\ell}\right)}
$$

because $r$ is unbiased and hence $m=n_{r}-1<n \cdot 2^{-\ell}+n \cdot 2^{-(2 k-\ell+1)} \leq 2 n \cdot 2^{-\ell}$ because $\ell<k$. Now, if $\mathrm{ra}$ were biased we would have

$$
\left|n_{r a}-n \cdot 2^{-(\ell+1)}\right|>n \cdot 2^{-(2 k-(\ell+1)+1)}
$$

Since $\left|n_{r a}-X\right| \leq 1$ and since $\left|\frac{1}{2} \cdot n_{r}-n \cdot 2^{-(\ell+1)}\right| \leq \frac{1}{2} \cdot n \cdot 2^{-(2 k-\ell+1)}$ because $r$ is unbiased, by the triangle inequality this would mean that

$$
\left|X-\frac{1}{2} \cdot n_{r}\right|>n \cdot 2^{-(2 k-(\ell+1)+1)}-\frac{1}{2} \cdot n \cdot 2^{-(2 k-\ell+1)}-1
$$

and in particular, using $0 \leq \ell \leq k-1$ and $4 k \leq \log _{2} n$, that

$$
\left|X-\frac{1}{2} \cdot\left(n_{r}-1\right)\right| \geq n \cdot 2^{-3 k} .
$$

The probability of this happening is bounded by (11) with $t=n \cdot 2^{-3 k}$, which is at most $\epsilon:=2 \exp (-n$. $\left.2^{-6 k}\right)$.

The argument is now finished by two union bounds. By the union bound over the $2^{\ell}$ leaves of $T_{\ell}$, the probability that some leaf of $T_{\ell}$ generates a biased child is at most $2^{\ell} \cdot \epsilon$. By the union bound over $\ell$, the probability that there exists an $\ell \in\{0, \ldots, k-1\}$ for which $\left(A_{\ell}^{\prime}\right)$ holds but $\left(A_{\ell+1}^{\prime}\right)$ fails is at most $\sum_{\ell=0}^{k-1} 2^{\ell} \cdot \epsilon$. Thus, the probability that $\left(A_{k}^{\prime}\right)$ fails is bounded by $2^{k+1} \cdot \exp \left(-n \cdot 2^{-6 k}\right)$.

Next we observe that $\left(A_{k}\right)$ implies $\left(B_{2^{k}}\right)$.

Lemma 12. For any graph $G$ and $k \geq 0$, if $\left(A_{k}\right)$ holds, then $\left(B_{2^{k}}\right)$ holds.

Proof. In a complete binary tree of depth $k$, the number of left children at depth at most $k$ is $\sum_{i=1}^{k} 2^{i-1}=$ $2^{k}-1$. Now, if $T_{k}$ satisfies $\left(A_{k}\right)$, then the root and every left child at depth at most $k$ has generated a proper step in the process of producing $T_{2^{k}}$; these are $2^{k}$ proper steps as claimed.

Finally we note that 3-element sets split with high probability if enough steps are proper. This is similar to Lemma 7 in [8].

Lemma 13. Let $G \sim G(n, 1 / 2)$ and let $k \geq 0$. Then, the probability that $\left(B_{k}\right)$ holds and $\left(C_{k}\right)$ fails is at most $\left(\begin{array}{l}n \\ 3\end{array}\right) \cdot 2^{-2 k}$.

Proof. Fix a 3-element set $A \subseteq V$ and fix $\ell \leq k$. Let $S_{\ell}$ denote the event that the set $A$ is not split at step $\ell$ and $P_{\ell}$ denote the event that step $\ell$ is proper. We aim to show that $\mathbb{P}\left[\cap_{\ell=1}^{k} S_{\ell} \cap \cap_{\ell=1}^{k} P_{\ell}\right] \leq 2^{-2 k}$ and the result then follows by a union bound over all three element subsets.

Now,

$$
\mathbb{P}\left[\bigcap_{\ell=1}^{k} S_{\ell} \cap \bigcap_{\ell=1}^{k} P_{\ell}\right]=\prod_{\ell=0}^{k-1} \mathbb{P}\left[S_{\ell+1} \cap P_{\ell+1} \mid \bigcap_{i=1}^{\ell}\left(S_{i} \cap P_{i}\right)\right]
$$

which is bounded by

$$
\prod_{\ell=0}^{k-1} \mathbb{P}\left[S_{\ell+1} \mid P_{\ell+1} \cap \bigcap_{i=1}^{\ell}\left(S_{i} \cap P_{i}\right)\right] .
$$

So, it suffices to show that each term in (12) is bounded by $\frac{1}{4}$. 
Fix $\ell \in\{0, \ldots, k-1\}$ and let $\mathbf{T}$ denote the sequence of splitting trees $T_{0}, \ldots, T_{\ell}$. Let $\mathcal{T}$ denote the set of all sequences of splitting trees of length $\ell+1$ and $\mathcal{T}_{A}$ denote the subset of $\mathcal{T}$ consisting of those sequences $\mathbf{U}=U_{0}, \ldots, U_{\ell}$ in which all steps are proper and $A$ does not split at any stage and $U_{\ell}$ splits properly, i.e. with respect to a node in $R^{\prime}\left(U_{\ell}\right)$. In other words, the sequence $\mathbf{U}$ satisfies $P_{\ell+1} \cap \bigcap_{i=1}^{\ell}\left(S_{i} \cap P_{i}\right)$. We now argue that, for any given $\mathbf{U} \in \mathcal{T}$, we have $\mathbb{P}\left[S_{\ell+1} \mid \mathbf{T}=\mathbf{U}\right] \leq \frac{1}{4}$.

Let $r$ be a leaf of $T_{\ell}$ such that $A \subseteq L_{r}$. Let $t$ be the node of $T_{\ell}$ with respect to which the splitting step $\ell+1$ is made. We argue that, conditioned on the event that this step is proper, i.e. $t$ belongs to $R^{\prime}\left(T_{\ell}\right)$, the probability that the elements of $A$ are not split apart in $T_{\ell+1}$ is at most $1 / 4$. Let $Y$ be the unique set in $\mathcal{S}\left(T_{\ell}\right)$ of which $L_{t}$ is a proper subset. By the discussion after Lemma 10, the tree $T_{\ell+1}$ can be seen as produced by first choosing $H$ uniformly at random among the graphs that are consistent with $T_{\ell}$, and then applying the splitting procedure on $T_{\ell}$ with respect to $t$ and $H$. By Lemma 10, the distribution of $\left\{p\left(H, x, L_{t}\right)\right\}_{x \in V}$ is uniform over the assignments that satisfy the constraints

$$
p\left(G, S, L_{t}\right)=\ell\left(L_{t}, S\right) .
$$

for every $S \in \mathcal{S}\left(T_{\ell}\right) \backslash\{Y\}$. In particular, since all sets in $\mathcal{S}\left(T_{\ell}\right)$ are pairwise disjoint, if $S$ is the unique minimal set in $\mathcal{S}\left(T_{\ell}\right)$ that contains $L_{r}$, then the distribution of $\left\{p\left(H, x, L_{t}\right)\right\}_{x \in S}$ is uniform over the assignments that satisfy the constraint (13) for this $S$ only, or no constraint at all if $S=Y$. Thus, in case $S \neq Y$ there are $2^{\left|S \cup L_{t}\right|-1}$ choices for $\left\{p\left(H, x, L_{t}\right)\right\}_{x \in S}$ and $2^{\left|S \cup L_{t}\right|-3}$ such choices that are constant over $A$, and in case $S=Y$ there are $2^{\left|S \cup L_{t}\right|}$ choices for $\left\{p\left(H, x, L_{t}\right)\right\}_{x \in S}$ and $2^{\left|S \cup L_{t}\right|-2}$ such choices that are constant over $A$. In both cases this gives probability $1 / 4$ as claimed.

To complete the argument, let $E_{\ell}$ denote the event $P_{\ell+1} \cap \bigcap_{i=1}^{\ell}\left(S_{i} \cap P_{i}\right)$. We have:

$$
\begin{aligned}
\mathbb{P}\left[S_{\ell+1} \mid E_{\ell}\right] & =\mathbb{P}\left[S_{\ell+1} \cap E_{\ell}\right] \cdot \mathbb{P}\left[E_{\ell}\right]^{-1} \\
& =\sum_{\mathbf{U} \in \mathcal{T}} \mathbb{P}\left[S_{\ell+1} \cap E_{\ell} \mid \mathbf{T}=\mathbf{U}\right] \cdot \mathbb{P}[\mathbf{T}=\mathbf{U}] \cdot \mathbb{P}\left[E_{\ell}\right]^{-1} \\
& =\sum_{\mathbf{U} \in \mathcal{T}_{A}} \mathbb{P}\left[S_{\ell+1} \mid \mathbf{T}=\mathbf{U}\right] \cdot \mathbb{P}[\mathbf{T}=\mathbf{U}] \cdot \mathbb{P}\left[E_{\ell}\right]^{-1} \\
& \leq \frac{1}{4} \cdot \sum_{\mathbf{U} \in \mathcal{T}_{A}} \mathbb{P}[\mathbf{T}=\mathbf{U}] \cdot \mathbb{P}\left[E_{\ell}\right]^{-1} \\
& =\frac{1}{4} \cdot \mathbb{P}\left[E_{\ell}\right] \cdot \mathbb{P}\left[E_{\ell}\right]^{-1} \\
& =\frac{1}{4} .
\end{aligned}
$$

This completes the proof of the lemma.

We are ready to synthesize what we have learned in a single lemma. In its statement, the choice of parameters is made to minimize the probability of failure. Other choices with other goals would work as well.

Lemma 14. Let $G \sim G(n, 1 / 2)$. Then, the probability that $T_{\left\lceil n^{1 / 5}\right\rceil}$ does not satisfy $\left(C_{\left\lceil n^{1 / 5}\right\rceil}\right)$ is at most $2^{-\Omega\left(n^{1 / 6}\right)}$.

Proof. Choose $k=\left\lceil\frac{1}{5} \log _{2} n\right\rceil$ in Lemma 11 and $k=\left\lceil n^{1 / 5}\right\rceil$ in Lemma 13 and link them through Lemma 12.

\subsection{Defining the splitting steps}

In this section we show that sets $L_{t}$ of the splitting trees $T_{k}$ are definable by formulas $\psi_{t}(x)$ of very low quantifier rank. First let us recall that if the splitting step is made with respect to node $t$, then every leaf $s$ 
splits into the sets

$$
\begin{aligned}
& L_{s 0}=\left\{x \in L_{s}: p\left(G, x, L_{t}\right)=0\right\} \\
& L_{s 1}=\left\{x \in L_{s}: p\left(G, x, L_{t}\right)=1\right\} .
\end{aligned}
$$

Note that the nodes at depth $\ell$ are generated by the $\ell$-th splitting step. For every non-root node $t$ in a splitting tree $T$, let $v_{T}(t)$ be the node of $T$ that generated $t$. In the following let $u(1):=1$ and $u(\ell):=\operatorname{bin}_{2}(2(\ell-1))$ for every $\ell \geq 2$.

Lemma 15. Let $G$ be a graph and let $k \geq \ell \geq 1$. Then, for every node $t$ at depth $\ell$ in $T_{k}$, we have $v_{T_{k}}(t)=u(\ell)$.

Proof. Let us write $T=T_{k}$. If $t$ is one of the two nodes at depth 1 , then $v_{T}(t)$ is the root, which agrees with $u(1)$. Assume now that $t$ is a node at depth $\ell \geq 2$. Let $\operatorname{num}_{2}$ be such that $\operatorname{num}_{2}\left(\operatorname{bin}_{2}(n)\right)=n$ for every positive integer $n$. We show that $\operatorname{num}_{2}\left(v_{T}(t)\right)=2(\ell-1)$. We proceed by induction on $\ell$. For $\ell=2$ we have it since then $v_{T}(t)$ is the left child of the root 10 , and $\operatorname{num}_{2}(10)=2$. Now, if $t$ is a node at depth $\ell \geq 2$ and we assume that $\operatorname{num}_{2}\left(v_{T}(t)\right)=2(\ell-1)$, then for every $a \in\{0,1\}$ we have

$$
\operatorname{num}_{2}\left(v_{T}(t a)\right)=\operatorname{num}_{2}\left(v_{T}(t)\right)+2=2(\ell-1)+2=2((\ell+1)-1)
$$

where the first follows from the fact that the nodes at level $\ell+1$ are generated by the next left-child following $v_{T}(t)$ in the level-order, and that the level-order on nodes agrees with the order of the natural numbers when they are read in binary.

Now, for $a_{1}, \ldots, a_{\ell} \in\{0,1\}$, define

$$
\psi_{1 a_{1} \cdots a_{\ell}}(x):=\bigwedge_{\substack{i=1 \\ a_{i}=1}}^{\ell} \oplus z\left(\psi_{u(i)}(z) \wedge E(x, z)\right) \wedge \bigwedge_{\substack{i=1 \\ a_{i}=0}}^{\ell} \neg \oplus z\left(\psi_{u(i)}(z) \wedge E(x, z)\right) .
$$

Note that $\psi_{1}(x)$ is true since then the conjunctions are empty. We show that the $\psi_{t}(x)$ are the formulas we are after.

Lemma 16. Let $G$ be a graph and let $k \geq \ell \geq 0$. Then, for every node $t$ at depth at most $\ell$ in $T_{k}$, the formula $\psi_{t}(x)$ defines the set $L_{t}$ in $G$.

Proof. For every non-leaf node $t$ at depth $\ell-1$ we have $v_{T}(t a)=u(\ell)$ for both $a=0$ and $a=1$ by Lemma 15. Therefore

$$
\begin{aligned}
& L_{t 0}=\left\{x \in L_{t}: p\left(G, x, L_{u(\ell)}\right)=0\right\} \\
& L_{t 1}=\left\{x \in L_{t}: p\left(G, x, L_{u(\ell)}\right)=1\right\},
\end{aligned}
$$

Now, if $t=1 a_{1} a_{2} \cdots a_{\ell}$, then unfolding the recursion we have that $L_{t}$ is the set of vertices $x \in V$ for which $p\left(G, x, L_{u(i)}\right)=a_{i}$ holds for every $i \in\{1, \ldots, \ell\}$. This is precisely what $\psi_{t}(x)$ says.

Note that the quantifier rank of $\psi_{t}(x)$ depends only on the depth of $t$. Therefore, let $q(\ell)$ be the quantifier rank of $\psi_{t}(x)$ for some and hence every $t$ of depth $\ell$. Note that $q(\ell)$ is monotone non-decreasing.

Lemma 17. $q(\ell)=O\left(\log ^{*} \ell\right)$.

Proof. If $t$ is a node at depth $\ell$, the largest $u(i)$ in the definition of $\psi_{t}$ is $2(\ell-1)$. Since $q$ is monotone non-decreasing we have

$$
q(\ell)=1+q\left(\left|\operatorname{bin}_{2}(2(\ell-1))\right|\right) .
$$

Since the length of $\operatorname{bin}_{2}(2(\ell-1))$ is $\log _{2}(\ell)+O(1)$, this recurrence gives $q(\ell)=O\left(\log ^{*} \ell\right)$ as claimed. 


\subsection{Defining the linear pre-order}

Finally we are ready to prove the main lemma of this section.

Lemma 18. There exists $d>0$ such that for every $\delta>0$, there exists $n_{0} \geq 0$ such that for every $n \geq n_{0}$ there is a formula $\psi(x, y)$ of quantifier rank at most $d \log ^{*} n$ such that, for $G \sim G(n, 1 / 2)$, the probability that $\varphi$ defines a linear pre-order of width at most 2 is at least $1-\delta$.

Proof. Choose $d$ to be the universal multiplicative constant in the $O\left(\log ^{*} \ell\right)$ notation in Lemma 17. Fix $\delta>0$ and let $n_{0}$ be large enough so that for every $n \geq n_{0}$ the probability in Lemma 14 is at most $\delta$, and $q(n) \leq d \log ^{*} n$. For fixed $n \geq n_{0}$, let $k=\left\lceil n^{1 / 5}\right\rceil$, and let $\psi(x, y)$ be the formula:

$$
\bigvee_{\substack{s, t \\ s \leq t}} \psi_{s}(x) \wedge \psi_{t}(y),
$$

where $s$ and $t$ range over the leaves of $T_{k}$ in the disjunction. If $T_{k}$ has all its leaves labeled by sets of size at most two this defines a linear pre-order of width at most two. By choice of $n_{0}$ this happens with probability at least $1-\delta$. Finally, by Lemma 17, the quantifier rank of $\psi$ is $q(k) \leq q(n) \leq d \log ^{*} n$.

\section{Establishing the lower bound}

Here we put it all together to prove Theorem 1.

Theorem 19. 1 There exists a constant $c>0$ such that for every large enough integer $q$, every $\epsilon>0$, and every large enough integer $n$, there exists an $F O[\oplus]$-formula $\phi(u, v, w)$ of quantifier rank $q$ such that, for every Boolean combination $p$ of quantifier-free formulas and $F O[\oplus]$-polynomials of degree bounded by a tower of exponentials of height at most $q / c$, the formulas $\phi$ and $p$ must disagree on a fraction $1-\epsilon$ of all graphs with $n$ vertices.

Proof. Let $d$ be the constant in Lemma 18 and choose $c:=d+1$. Choose $q_{0}$ large enough (to be determined later), fix $q \geq q_{0}$ and $\epsilon>0$, and choose $n_{0}$ large enough (to be determined later) and fix $n \geq n_{0}$. Let $\psi(x, y)$ be the formula from Lemma 18 for $\delta=\epsilon / 2$ and the fixed $n$; in particular $n_{0}$ must be larger than the $n_{0}$ from Lemma 18 for this particular $\delta$. Let $\phi(x, y, z):=\psi(x, y) \wedge \psi(y, z)$. Since we want the quantifier rank of $\phi$ to be at most $q$, we choose $n_{0}$ large enough so that $d \log ^{*} n \geq q$ whenever $n \geq n_{0}$. We claim that this $\phi(x, y, z)$ witnesses the theorem.

Suppose $p(x, y, z)$ is a Boolean combination of quantifier-free formulas and $\mathrm{FO}[\oplus]$-polynomials of degree bounded by a tower of exponentials of height $q / c$ that agrees with $\phi(x, y, z)$ on more than an $\epsilon$ fraction of graphs with $n$ vertices. Since $q \geq q_{0}$ is large enough, a tower of exponentials of height $q / c=$ $q /(d+1)$ has height at most $q / d-4$, and since $q \leq d \log ^{*} n$, the degree is bounded by $\log \log \log \log n$. By Remarks 1 and 2 we may assume that $p(x, y, z)$ is a Boolean combination of $\mathrm{FO}[\oplus]$-polynomials of order $2 \log \log \log \log n+1$, which is at most $\log \log \log n$ if $n \geq n_{0}$ is large enough.

By Lemma 2 we may assume that $p(x, y, z)$ is a regular normal form of this order. If $n_{0}$ is large enough, with probability at least $1-\epsilon / 2$ there exists a triple $a, b, c$ of distinct vertices for which $Y(a, b, c)$ holds. Also if $n_{0}$ is large enough, with probability at least $1-\epsilon / 2$ the formula $\psi(x, y)$ defines a linear pre-order of width at most 2 . By the union bound, with positive probability all three hold:

1. $\phi(x, y, z)$ and $p(x, y, z)$ agree on $G$,

2. $\psi(x, y)$ defines a linear pre-order $\preceq$ of width at most 2 in $G$, 
3. there exists a triple of distinct vertices $a, b, c$ of $G$ for which $Y(a, b, c)$ holds.

Now, assume without loss of generality that $a \preceq b \preceq c$ : otherwise permute them accordingly. Note that we cannot have $c \preceq a$ as otherwise all three $a, b, c$ would belong to the same class of the pre-order, which is not possible because its width is 2 and $a, b, c$ are distinct. But then $\phi(a, b, c)$ holds and $\phi(c, a, b)$ does not hold, which means that $\phi$ distinguishes one permutation of $(a, b, c)$ from another. But then $p$ also does; a contradiction to $Y(a, b, c)$.

Final remarks The lower bound is achieved by a formula with free variables. In particular, when we say that $p(x, y, z)$ cannot agree with $\phi(x, y, z)$ on more than an $\epsilon$-fraction of the graphs with $n$ vertices, what we mean is that, on at least a $(1-\epsilon)$-fraction of the graphs, the ternary relations on the set of vertices that are defined by $\phi(x, y, z)$ and $p(x, y, z)$ are not identical. It would be nice to obtain a similar kind of lower bound for sentences, i.e. formulas without free variables. However, since every sentence $\phi$ agrees on at least half the graphs with $n$ vertices with one of the two constant polynomials $p=0$ or $p=1$, the correct lower-bound statement in this setting is different: that for every $\epsilon>0$ and every sufficiently large $n$, any low-degree $p$ cannot agree with $\phi$ on more than a $\left(\frac{1}{2}+\epsilon\right)$-fraction of the graphs with $n$ vertices. A candidate such sentence $\phi$ could be the one saying that the number of edges between the minimum and the maximum classes in the pre-order is odd. However we were not able to prove that this sentence $\phi$ must be uncorrelated to any low degree $\mathrm{FO}[\oplus]$-polynomial $p$, i.e. that for any such $p$, the probability that $\phi$ and $p$ agree on a random graph is very close to $1 / 2$. We leave this as an interesting open problem.

Acknowledgment We are grateful to Swastik Kopparty for discussions and comments on a previous version of this paper.

\section{References}

[1] E. Allender. A Note on the Power of Threshold Circuits. In Proceedings of the 30th Symposium on Foundations of Computer Science (FOCS), pages 580-584, 1989.

[2] R. Beigel and J. Tarui. On ACC. Computational Complexity, 4:350-366, 1994.

[3] K. J. Compton. 0-1 Laws in Logic and Combinatorics. In I. Rival, editor, NATO Advanced Study Institute on Algorithms and Order, pages 353-383. Kluwer, 1989.

[4] A. Dawar and E. Grädel. Properties of Almost All Graphs and Generalized Quantifiers. Fundameta Informaticae, 98(4):351-372, 2010.

[5] R. Fagin. Probabilities on Finite Models. Journal of Symbolic Logic, 41(1):50-58, 1976.

[6] Y. V. Glebskiǔ, D. I. Kogan, M. I. Ligon'kiǔ, and V. A. Talanov. Range and Degree of Realizability of Formulas in the Restricted Predicate Calculus. Kibernetika, 2:17-28, 1969.

[7] L. Hella, P. G. Kolaitis, and K. Luosto. Almost everywhere equivalence of logics in finite model theory. Bulletin of Symbolic Logic, 2:422-443, 1996.

[8] R. M. Karp. Probabilistic Analysis of a Canonical Numbering Algorithm for Graphs. In Proceedings of the AMS Symposium in Pure Mathematics, volume 34, pages 365-378, 1979. 
[9] Ph. G. Kolaitis and S. Kopparty. Random Graphs and the Parity Quantifier. In Proceedings of the 41st ACM Symposium on the Theory of Computing (STOC), pages 705-714, 2009.

[10] Ph. G. Kolaitis and S. Kopparty. Random Graphs and the Parity Quantifier. Technical Report 33(2009), Electronic Colloquium on Computational Complexity (ECCC), 2009.

[11] Ph. G. Kolaitis and M. Y. Vardi. Infinitary Logics and 0-1 Laws. Information and Computation, 98(2):258-294, 1992.

[12] A. A. Razborov. Lower Bounds on the Size of Bounded Depth Networks over a Complete Basis with Logical Addition. Mathematical Notes of the Academy of Sciences of the USSR, 41:333-338, 1987.

[13] R. Smolensky. Algebraic Methods in the Theory of Lower Bounds for Boolean Circuit Complexity. In Proceedings of the 19th ACM Symposium on the Theory of Computing (STOC), pages 77-82, 1987.

[14] R. Williams. Non-uniform ACC Circuit Lower Bounds. In Proceedings of the 26th IEEE Conference on Computational Complexity (CCC), pages 115-125, 2011. 